\title{
Ionospheric control of the dawn-dusk asymmetry of the Mars magnetotail current sheet
}

Michael W. Liemohn ${ }^{1}$, Shaosui $\mathrm{Xu}^{2}$, Chuanfei Dong ${ }^{3}$, Stephen W. Bougher ${ }^{1}$, Blake C. Johnson ${ }^{1}$, Raluca Ilie ${ }^{1,4}$, and Darren L. De Zeeuw ${ }^{1}$

${ }^{1}$ Department of Climate and Space Sciences and Engineering, University of Michigan, Ann Arbor, MI.

${ }^{2}$ Space Sciences Laboratory, University of California, Berkeley, CA.

${ }^{3}$ Department of Astrophysical Sciences and Princeton Plasma Physics Laboratory, Princeton University, Princeton, NJ.

${ }^{4}$ Department of Electrical and Computer Engineering, University of Illinois, Urbana-Champaign, IL.

Corresponding author: Michael Liemohn (liemohn@umich.edu)

Submitted to the Journal of Geophysical Research - Space Physics, special section on Observations, Simulations and Theory of Electric Currents in the Solar System

\section{Key Points:}

- There is a systematic Y (i.e., dawn-dusk) asymmetry in the location of the Martian magnetotail current sheet in modified MSE coordinates

- The asymmetry is controlled by ionospheric conditions, shifting to the dawn (-Y) during solar maximum and to the dusk during solar minimum

- The shift found in this study is not a function of crustal fields, which were omitted, or solar wind conditions, which were held constant

AGU Index Terms:

- 2756 Planetary magnetospheres

- 2744 Magnetotail

- $5443 \quad$ Magnetospheres

This is the author manuscript accepted for publication and has undergone full peer review but has not been through the copyediting, typesetting, pagination and proofreading process, which may lead to differences between this version and the Version of Record. Please cite this article as doi: $10.1002 / 2016 \mathrm{JA} 023707$

This article is protected by copyright. All rights reserved. 
- 2721

Field-aligned currents and current systems

- 2780

Solar wind interactions with unmagnetized bodies

\section{Keywords:}

Mars magnetotail, Mars current sheet, solar wind-magnetosphere interaction, magnetized ionosphere

$=$
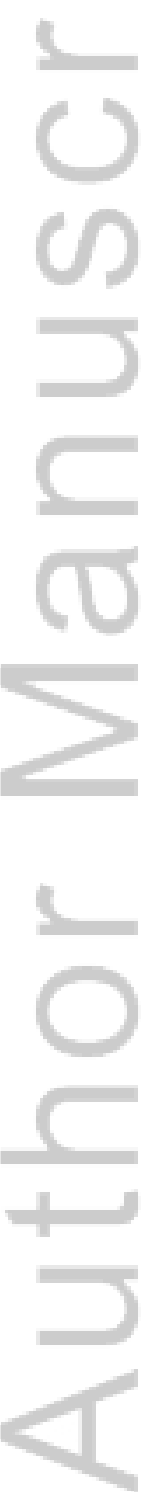

This article is protected by copyright. All rights reserved. 


\begin{abstract}
This study investigates the role of solar EUV intensity at controlling the location of the Mars magnetotail current sheet and the structure of the lobes. Four simulation results are examined from a multifluid magnetohydrodynamic model. The solar wind and interplanetary magnetic field (IMF) conditions are held constant and the Mars crustal field sources are omitted from the simulation configuration. This isolates the influence of solar EUV. It is found that solar maximum conditions, regardless of season, result in a Venus-like tail configuration with the current sheet shifted to the $-Y$ (dawnside) direction. Solar minimum conditions result in a flipped tail configuration with the current sheet shifted to the $+Y$ (duskside) direction. The lobes follow this pattern, with the current sheet shifting away from the larger lobe with the higher magnetic field magnitude. The physical process responsible for this solar EUV control of the magnetotail is the magnetization of the dayside ionosphere. During solar maximum, the ionosphere is relatively strong and the draped IMF field lines quickly slip past Mars. At solar minimum, the weaker ionosphere allows the draped IMF to move closer to the planet. These lower altitudes of the closest approach of the field line to Mars greatly hinders the day-to-night flow of magnetic flux. This results in a buildup of magnetic flux in the dawnside lobe as the $S$ shaped topology on that side of the magnetosheath extends farther downtail. The study demonstrates that the Mars dayside ionosphere exerts significant control over the nightside induced magnetosphere of that planet.
\end{abstract}

\title{
Plain language summary:
}

Mars, which does not have a strong magnetic field, has an induced magnetic environment from the draping of the interplanetary magnetic field from the Sun. It folds around Mars, forming two "lobes" of magnetic field behind the planet with a current sheet of electrified gas (plasma) behind it. The current sheet is not directly behind the planet but rather shifted towards the dawn or dusk direction. It is shown here that one factor controlling the location of the current sheet is the dayside ionosphere. At solar maximum, the ionosphere is dense, the magnetic field slips easily by the planet, and the current sheet is shifted toward dawn. At solar minimum, the ionosphere is relatively weak, the magnetic field slippage is slowed down, and the current sheet shifts toward dusk.

\section{Introduction}

Like Venus, Mars does not have a strong internal dipole magnetic field, so the solar wind plasma and interplanetary magnetic field (IMF) impinge directly on the planet's ionosphere [see reviews by, e.g., Luhmann and Brace, 1991; Nagy et al., 2004; Bertucci et al., 2011]. The draped IMF is stagnated in the planetary sheath region and, sometimes, the ionosphere. This results in two magnetic lobes behind the planet, between which flows a current of escaping 
planetary ions [e.g., Dubinin et al., 2006, 2011; Carlsson et al., 2006, 2008; Barabash et al., 2007; Fang et al., 2008; Dubinin and Fraenz, 2015].

An interesting feature of the magnetotail at Venus, seen in satellite observations, is that its location relative to the planet exhibits a dawn-to-dusk asymmetry, specifically shifted towards dawn [McComas et al., 1986]. This shift was confirmed by magnetohydrodynamic (MHD) calculations of the Venus space environment [Ma et al., 2013]. The reason for this asymmetry in the Venus tail is that the IMF, in its standard Parker spiral configuration from the $+\mathrm{X} /-\mathrm{Y}$ quadrant to the $-\mathrm{X} /+\mathrm{Y}$ quadrant (in planet-centered solar orbital coordinates), develops an $\mathrm{S}$ shaped topology in and near the planetary equatorial plane [see, e.g., Figure 3 of Liemohn et al., 2006]. The induced curvature on the $-Y$ dawnside results in the sheath region exerting less force on the magnetotail on the dawnside than the duskside. This sets up a smaller lobe on the dawnside and the current sheet shifts in the $-\mathrm{Y}$ direction [see, e.g., Figure 1 of McComas et al., 1986].

At Mars, this shift has also been observed. For instance, Halekas et al. [2006] found many current sheet crossings in upper ionospheric magnetic data at 2 AM local time, implying a systematic -Y shift in the magnetotail current sheet. In contrast, DiBraccio et al. [2015] showed observations of a satellite pass in the near-Mars tail, showing a shift to the $+Y$ direction for the magnetic field reversal (the location of the current sheet).

Published studies examining MHD simulations of the Mars space environment are not conclusive in explaining this issue. Harnett and Winglee [2005] found a similar dawnward shift of the current sheet in single-fluid MHD simulation results. However, Ma et al. [2002, 2004], with a multi-species MHD model (separate continuity equations for each ion species but a single combined momentum and energy equation), found an opposite shift, with the current sheet shifted to the $+Y$. Similarly, Xu et al. [2016] examined output from a multifluid MHD simulation of the Mars space environment and found a tail current sheet shift to the $+Y$ direction. On the other hand, the multifluid simulation results presented and discussed in Najib et al. [2011] and the multi-species run analysis of Luhmann et al. [2015] revealed a -Y position of the current sheet. Finally, Li et al. [2013] show more of a rotation of the tail current sheet, also from multispecies MHD simulations. While there are differences between these studies, all of these simulations were set up for solar maximum conditions and nominal solar wind upstream input. In addition, all of these simulations included the crustal fields.

One confounding element in this issue is the presence of the crustal fields. Several studies have found systematic influences of the crustal field locations on magnetotail configurations [e.g., Ma et al., 2002; Harnett and Winglee, 2005; Fang et al., 2010, 2015; Dong et al., 2015a]. Luhmann et al. [2015] concludes that, perhaps, much of the magnetotail is connected to the planet rather than being IMF field lines draped around the planet.

This study simplifies the examination of the question by removing the crustal magnetic field from the MHD simulation set up. Several simulations are presented to address the question 
of what else besides the crustal fields, if anything, might control the location of the tail current sheet location in the $\mathrm{Y}$ coordinate.

\section{Model Setup}

This study uses simulation results from the Block-Adaptive-Tree Solar-wind Roe-type Upwind Scheme (BATS-R-US) multifluid magnetohydrodynamic (MF-MHD) model, as presented in detail by Najib et al. [2011] and Dong et al. [2014]. The plasma flow through the near-Mars space environment is solved with a combination of continuity, momentum, and energy equations for four ion species $\left(\mathrm{H}^{+}, \mathrm{O}^{+}, \mathrm{O}_{2}{ }^{+}\right.$, and $\mathrm{CO}_{2}{ }^{+}$, along with an induction equation for the local magnetic field. The grid extends from the bottom of the ionosphere at $100 \mathrm{~km}$ altitude to 8 $\mathrm{R}_{\mathrm{M}}$ upstream, $24 \mathrm{R}_{\mathrm{M}}$ downstream, and $16 \mathrm{R}_{\mathrm{M}}$ to each side. The spherical grid has a $1.5^{\circ}$ angular resolution near the planet, switching to $3^{\circ}$ resolution away from the planet. The radial grid is nonuniform, continually varying from $5 \mathrm{~km}$ at the inner boundary to $1 / 4 \mathrm{R}_{\mathrm{M}}$ near the outer boundary. Inclusion of chemistry allows for production and loss throughout the ionosphere. The thermospheric values are taken from the Mars Global Ionosphere Thermosphere Model [Bougher et al., 2015] and the hot coronal densities are taken from the Mars Adaptive Mesh Particle Simulator [Lee et al., 2015]. Note that both the thermosphere and exosphere are 3D simulation results and therefore include asymmetries due to the chemistry and physics of the upper atmosphere, as shown in Dong et al. [2015a].

The primary difference from previous simulation configurations is that the crustal fields are not included. The inner boundary is set with $\mathbf{B}=0$ at the innermost face of the inner shell of cells (that is, right at $100 \mathrm{~km}$ altitude). The velocity inner boundary condition is set to be reflective, such that the first ghost cell (that is, the buffer cell centered at $97.5 \mathrm{~km}$ altitude) has its velocity updated to have the negative radial velocity component of the first true cell and the same horizontal velocity components as the first true cell. The $\mathrm{O}^{+}, \mathrm{O}_{2}{ }^{+}$and $\mathrm{CO}_{2}{ }^{+}$are set at lower boundary based on photochemical equilibrium. The $\mathrm{H}^{+}$is set as 0.3 times the solar wind proton density. This leads to densities for the four species that are rather small values in the ghost cells and do not influence the overall result. Following Dong et al. [2014, 2015a], the grid resolution is most refined near the inner boundary of the MHD simulation domain, with a $5 \mathrm{~km}$ altitude grid spacing and $1.5^{\circ}$ angular resolution. The radial step size slowly changes with altitude to become $1000 \mathrm{~km}$ at the outer boundary $\left(\sim 20 \mathrm{R}_{\mathrm{M}}\right)$ and the angular resolution goes through a factor of two coarsening at about $1 \mathrm{R}_{\mathrm{M}}$ altitude to a $3^{\circ}$ grid size in both polar and azimuthal angle.

Upstream solar wind conditions are set with a proton density of $4 \mathrm{~cm}^{-3}$, velocity of 400 $\mathrm{km} / \mathrm{s}$ in the $-X$ direction, and a temperature of $3.5 \times 10^{5} \mathrm{~K}$. The IMF applied is a nominal away sector Parker spiral, with an angle $56^{\circ}$ off of the $-X$ axis and a magnitude of 3 nT. The quantities of $\mathrm{Vy}, \mathrm{Vz}$, and $\mathrm{Bz}$ are set to zero at the upstream boundary. The downstream boundaries are specified with a von Neumann zero slope but floating value boundary condition. For more information about the boundary settings and code implementation, please see Powell et al. [1999] for the numerical implementation of the 8-wave scheme, Gombosi et al. [2002] for the 
semi-relativistic equation set and numerical convergence algorithm, and Dong et al. [2014, 2015a] for the latest Mars multi-fluid code configuration.

The one thing that is varied between the simulations presented below is the solar EUV input to the model, which changes the photoionization rate, and the assumed neutral atmosphere distributions. Two simulations are conducted with solar maximum atmospheric and EUV conditions, at perihelion and aphelion, respectively, and two are conducted for solar minimum atmospheric and EUV conditions at these same two orbital locations. The third column of Table 1 lists the specific EUV flux intensity for each simulation, as defined by the proxy of the translated-to-Mars F10.7 value (in solar flux units, $10^{-22} \mathrm{~W} \mathrm{~m}^{-2} \mathrm{~Hz}^{-1}$ ). The lowest is $26 \mathrm{sfu}$ and the highest is $104 \mathrm{sfu}$, a variation of a factor of 4 . The seasonal change in Mars-F10.7 is roughly $40 \%$, while the solar cycle change is nearly a factor of three. These four settings span the full range of expected EUV input levels that are typical at Mars.

Table 1. Key Simulation Input and Output Values

\begin{tabular}{|c|c|c|c|c|c|c|c|c|c|}
\hline $\begin{array}{c}\text { Solar } \\
\text { Cycle }\end{array}$ & Orbital & $\begin{array}{c}\text { Mars } \\
\text { Location } \\
\text { Value }\end{array}$ & $\begin{array}{c}\text { Peak }|\mathrm{B}| \\
\text { in the } \\
\text { pileup } \\
\text { region }\end{array}$ & $\begin{array}{c}\text { Current } \\
\text { sheet Y } \\
\text { value at } \\
\mathrm{X}=-4 \mathrm{R}_{\mathrm{M}} \\
\& \mathrm{Z}=0\end{array}$ & $\begin{array}{c}\text { Current } \\
\text { sheet } \\
\mathrm{By} \text { at } \\
(\mathrm{X}, \mathrm{Z})= \\
(-4,0)\end{array}$ & $\begin{array}{c}\text { Current } \\
\text { sheet Jz at } \\
(\mathrm{X}, \mathrm{Z})= \\
(-4,0)\end{array}$ & $\begin{array}{c}\text { Integral of } \\
|\mathrm{Br}| \text { at } 120 \\
\mathrm{~km} \\
\text { altitude }\end{array}$ & $\begin{array}{c}\text { Peak } \mathrm{B}_{\text {in }} \\
\text { at } 120 \\
\mathrm{~km} \\
\text { altitude }\end{array}$ & $\begin{array}{c}\text { Peak } \\
\mathrm{B}_{\text {out }} \text { at } \\
120 \mathrm{~km} \\
\text { altitude }\end{array}$ \\
\hline Max & Perihelion & $104 \mathrm{sfu}$ & $49.6 \mathrm{nT}$ & $-0.36 \mathrm{R}_{\mathrm{M}}$ & $0.15 \mathrm{nT}$ & $5.8 \mathrm{nA} / \mathrm{m}^{2}$ & $\begin{array}{c}2.04 \times 10^{5} \\
\mathrm{~Wb}\end{array}$ & $6.1 \mathrm{nT}$ & $5.3 \mathrm{nT}$ \\
\hline Max & Aphelion & 74 & 51.1 & -0.04 & 0.35 & 6.1 & $2.02 \times 10^{5}$ & 7.4 & 4.1 \\
\hline Min & Perihelion & 36 & 54.2 & 1.04 & 0.52 & 6.6 & $2.18 \times 10^{5}$ & 10.3 & 5.3 \\
\hline Min & Aphelion & 26 & 54.1 & 0.84 & 0.92 & 6.0 & $2.47 \times 10^{5}$ & 15.1 & 7.7 \\
\hline
\end{tabular}

\section{Results}

\subsection{Global view}

Figure 1 presents an overview of the simulation results for the 4 cases. The panels in Figure 1 show identical 3D views from the afternoon sector above the equatorial plane, with $|\mathrm{B}|$ shown as a color background on two slices through the simulation result, at $Z=0$ (the equatorial plane) and $X=-4 R_{M}$. The small black dots on the axes are $1 R_{M}$ apart. To focus on the field in the lobes, the colorscale for $|\mathrm{B}|$ saturates at $25 \mathrm{nT}$, but values in the dayside pile-up region peak at larger values, as listed in the fourth column of Table 1. It should be noted that the solar maximum peak $|\mathrm{B}|$ values are near $50 \mathrm{nT}$ while the solar minimum peak $|\mathrm{B}|$ values are slightly higher, near 54 nT. Magnetic field traces are shown on each plot of Figure 2, initiated from 
manually chosen locations in the $X=-4 R_{M}$ plane. Six of the field-line traces were started from the dawn-side lobe in the $Y \sim-2.5$ to $-3 R_{M}$ region, while another 6 traces were started from the dusk-side lobe in the $\mathrm{Y} \sim 1.5$ to $2 \mathrm{R}_{\mathrm{M}}$ region. All 12 starting points in the $\mathrm{X}=-4 \mathrm{R}_{\mathrm{M}}$ plane are the same in the 4 panels. The coloring on the field line traces is the local altitude of the field line, with a scale that saturates at $600 \mathrm{~km}$ to focus on the parts of the field lines in the dayside pile-up region.

The location of the current sheet is readily seen in the magnitude of the magnetic field, which is presented in Figure 1 for the four cases. Magnetic field magnitude, rather than current density, is selected because it not only reveals the location of the current sheet (the magnetic field minimum) but also shows the location and magnitude of the lobes on either side of the current sheet. The location of the tail current sheet is evident in all of the panels of Figure 1 as the blue streak extending behind the planet between the green/yellow lobes. In the solar maximum cases (top row), the current sheet is located at midnight $(\mathrm{Y} \sim 0)$ or slightly towards dawn (-Y direction). In the solar minimum cases (bottom row), the current sheet is highly skewed to the $+\mathrm{Y}$ duskward direction, by nearly a planetary radius. The exact $\mathrm{Y}$ values of the minimum $B$ location at $X=-4 R_{M}$ and $Z=0$ is listed in the fifth column of Table 1 and the values of By and $\mathrm{JZ}$ at this location are listed in columns 6 and 7 of Table 1. The dawn-dusk shift follows a similar pattern in the relative sizes of the two lobes. Specifically, the current sheet is offset in the direction of the smaller lobe. At solar maximum, the two lobes are nearly symmetric with the dusk lobe very slightly larger than the dawn lobe. For the solar minimum results, the reverse is true and the dawn lobe is significantly larger than the dusk lobe.

This article is protected by copyright. All rights reserved. 
(a) SolarMax@ Perihelion

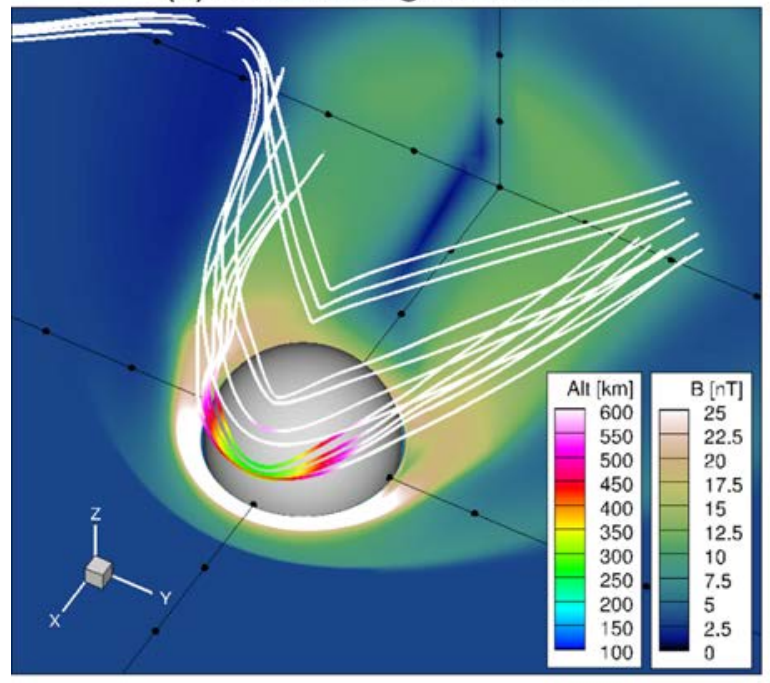

(c) Solar Min @ Perihelion

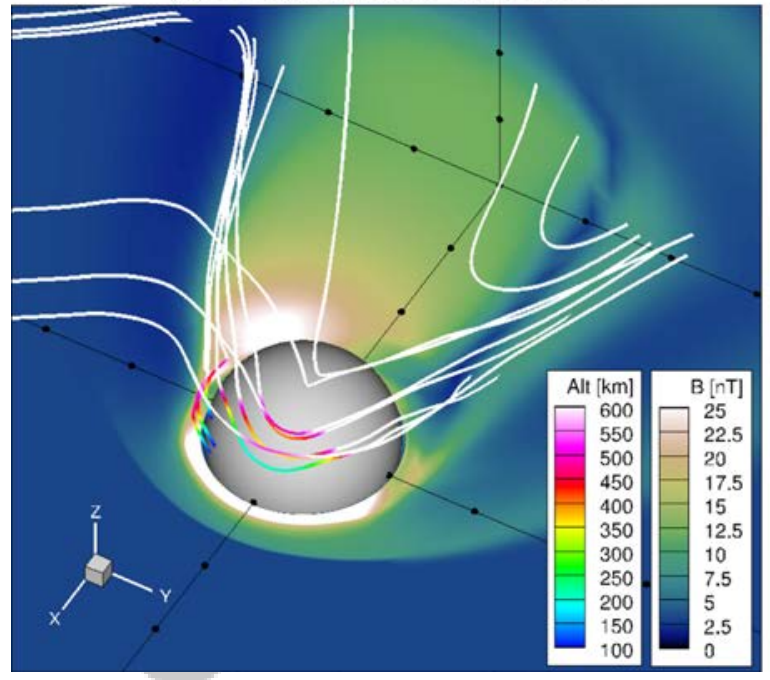

(b) Solar Max @ Aphelion

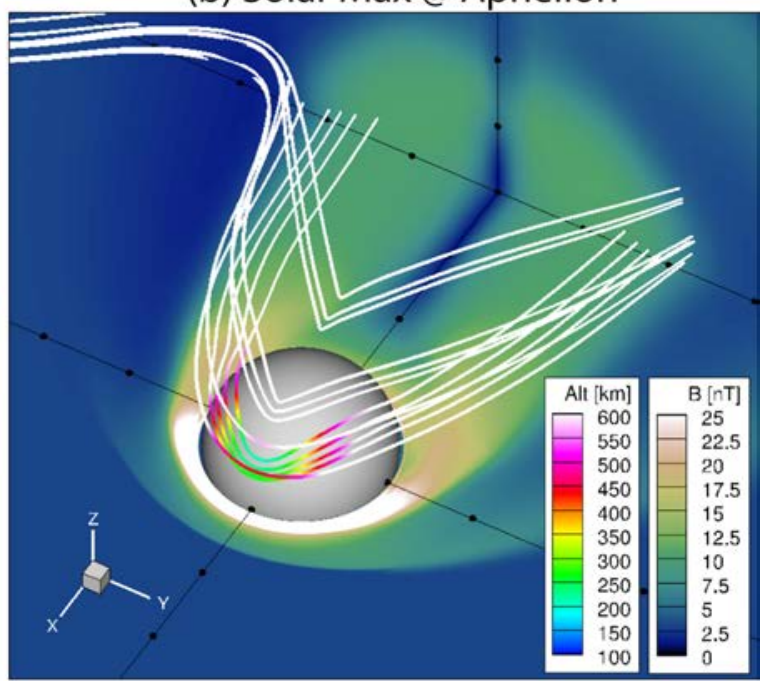

(d) Solar Min @ Aphelion

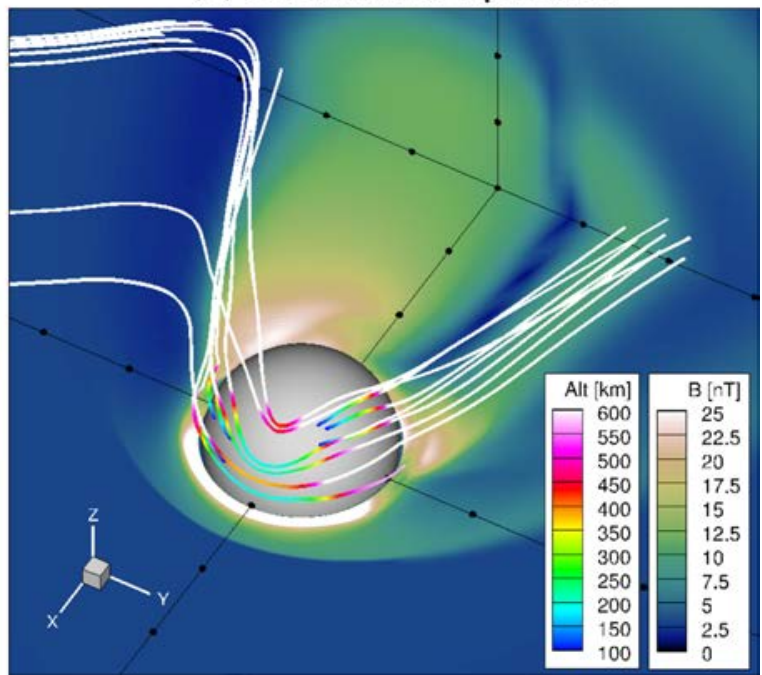

Figure 1. Model output of magnetic field intensity in the equatorial plane and the $X=-4 R_{M}$ plane (blue-green-pink color background) with a few representative 3D traces of magnetic field lines with altitude shown along the trace (lines with the bright rainbow color scale). The gray sphere is drawn at Mars' surface. The view is from above the ecliptic plane in the afternoon sector with the Sun down and to the left (axes legend is in the upper right of each plot). The dots along each axis are spaced $1 \mathrm{R}_{\mathrm{M}}$ apart.

A key feature of Figure 1 is the altitude of closest approach for the dozen representative 
field lines traced through the simulation results. For the two solar maximum runs (Figures 1a and $1 \mathrm{~b}$ ), a few of the field lines have slipped past the planet and include a sharp V-shaped kink at the place where they pass through the tail current sheet. These are colored white along their entire length because they remain above $600 \mathrm{~km}$ altitude everywhere. These field lines have the S-shaped configuration with the extra kink in the dawn sheath in order for the field line to bend back toward the planet before making another bend/kink to become part of the dusk lobe. Closer to the planet but in the far northern latitudes, a few of the field lines are seen to exhibit a weathervaning geometry as part of the field line was slowed down within the magnetic pileup region (MPR). These are still colored all white because they have already slipped through the lower-altitude pileup region and the slowed-down portion of the field lines are now being reaccelerated towards the tail. On the dayside at midlatitudes, the remainder of the field lines are shown to pass through the MPR, colored by altitude as they reach their closest approach to Mars. For the solar maximum results, the closest altitude for any of the chosen lines is between 250 and $300 \mathrm{~km}$.

For the two solar minimum runs, the paths of the representative field lines are somewhat different. In the perihelion solar minimum case (Figure 1c), there are still a few field lines that stay at "high" altitude (i.e., above the top of the color scale of $600 \mathrm{~km}$ ), while most of the field lines are colored for a portion of the trace, indicating relative closeness to the planet. Some of the lines, in fact, stop. These lines have reached the inner boundary of the simulation domain. That is, they have an "open" rather than "draped" topology. This is occurring even though there is no crustal fields in this simulation and the inner boundary is set to $\mathbf{B}=0$. In the aphelion solar minimum case (Figure 1d), all of the chosen field lines have some coloring along them, indicating that all of these field lines are still well within the MPR. There are even more lines that strike the inner boundary of the simulation domain and stop their trace before completing the bend around the dayside of Mars. For solar minimum, regardless of season and despite the inner boundary setting, some field lines are becoming open rather than remaining draped.

To explore this magnetic topology in more detail, Figure 2 presents a closer view from the vantage point of the morning sector (again, from the above the equatorial plane). The field lines are exactly the same as those shown in Figure 1, except that now they are colored by the local magnitude of velocity (i.e., speed), using the weighted average of velocities from all species in the simulation. The color scale for $|\mathrm{V}|$ is saturated at $40 \mathrm{~km} / \mathrm{s}$ to focus on the slowmoving plasma in the ionosphere and MPR. A small gap is seen between the gray sphere (Mars, drawn at $R=1 R_{M}$ ) and the equatorial plane of the MHD results (showing magnetic field magnitude, as in Figure 1). This is because the planar values start at the inner boundary of the simulation domain, at $100 \mathrm{~km}$ altitude. The black dots along the axes are shown every $0.1 \mathrm{R}_{\mathrm{M}}$, for reference.

Figure 2 shows that all four simulation results have at least some of the chosen field lines moving at speeds less than $2 \mathrm{~km} / \mathrm{s}$ (bottom of the blue scale). A speed of $2 \mathrm{~km} / \mathrm{s}$ translates to a field line motion of one Mars radius every 28 minutes, much slower than the $7 R_{M}$ per minute of 
the imposed solar wind. While these particular field lines do not precisely show a time sequence, they are from a steady state solution so a progression between some of them can be envisioned. At solar maximum (Figures $2 \mathrm{a}$ and $2 \mathrm{~b}$ ), the speeds pick up again within a radian of polar angle. That is, for solar maximum most the field lines are "very slow" $(<2 \mathrm{~km} / \mathrm{s})$ for less than one Mars radius. At solar minimum (Figures $2 \mathrm{c}$ and $2 \mathrm{~d}$ ), they are slow for a longer interval. Another feature of the solar minimum panels is the field line connection to the inner boundary of the simulation domain.

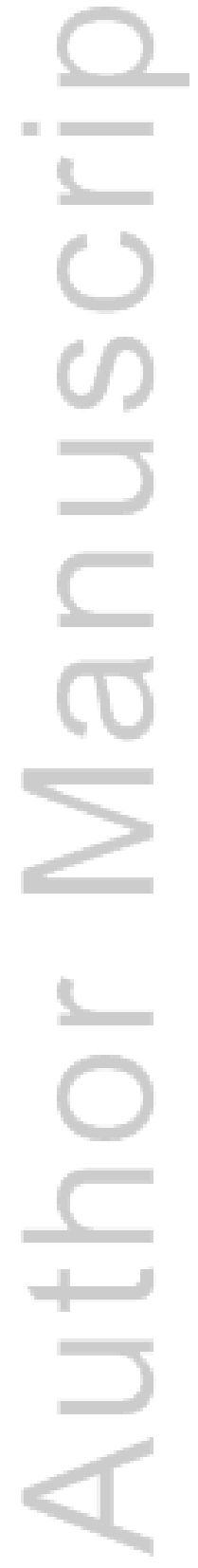


(a) Solar Max @ Perihelion

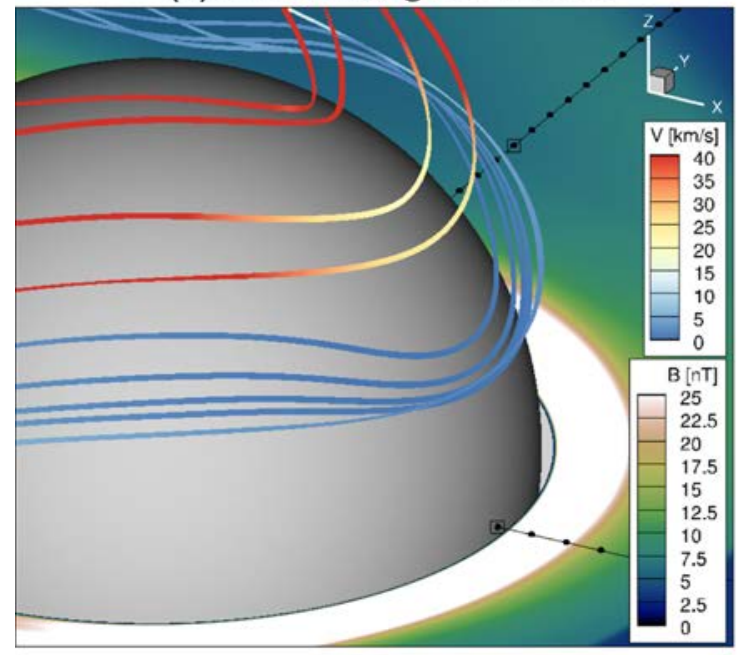

(c) Solar Min @ Perihelion

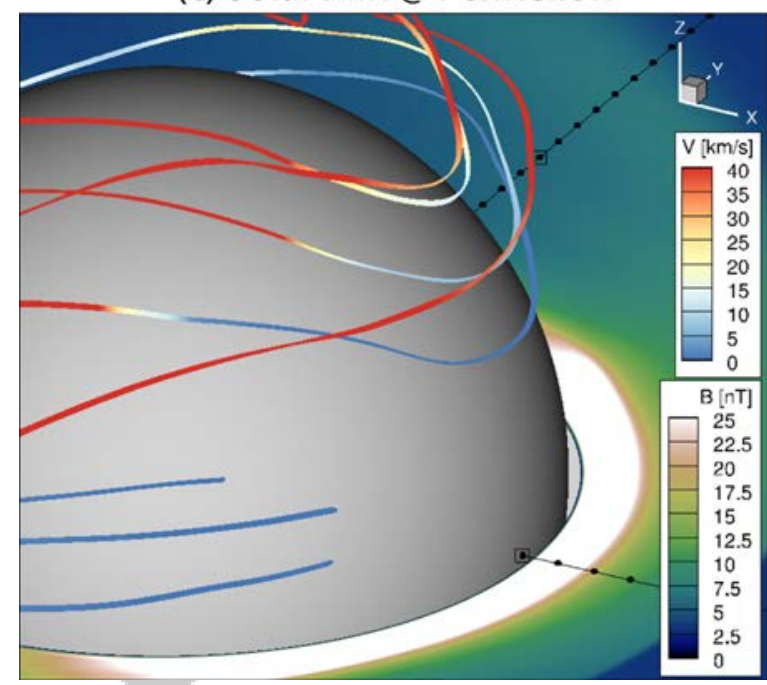

(b) Solar Max @ Aphelion

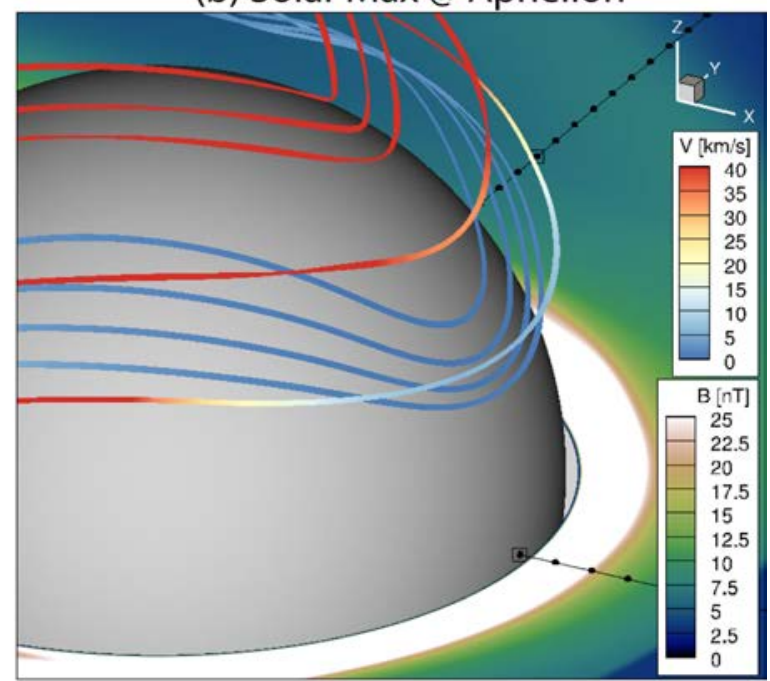

(d) Solar Min @ Aphelion

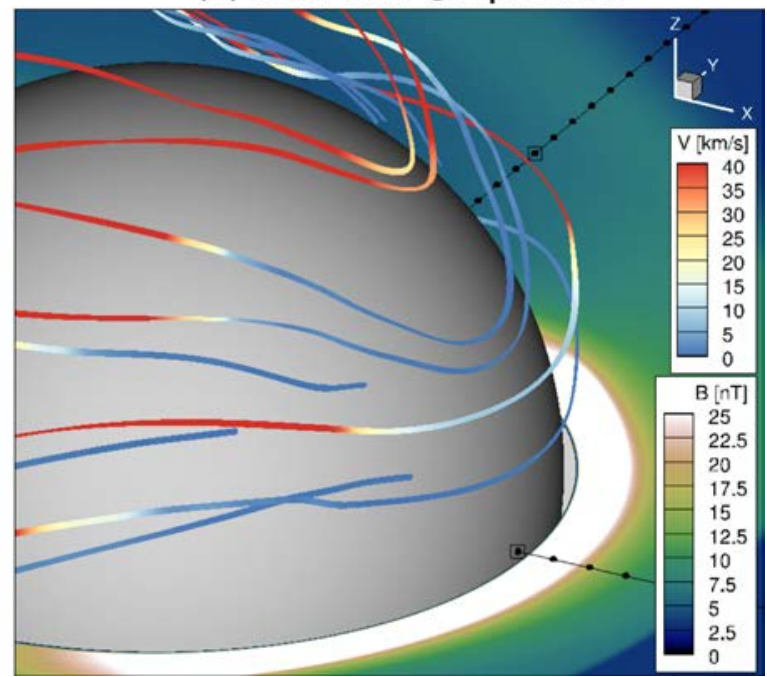

Figure 2. A view from the morning sector above the equatorial plane, zooming in on the field line traces through the dayside pileup region. Shown is magnetic field intensity in the equatorial plane (blue-green-pink color background, same scale as Figure 1) with the same representative 3D traces of magnetic field lines as Figure 1. This time, however, the field lines are colored with the local speed (magnitude of the velocity vector, blue-yellow-red color scale). The gray sphere is drawn at the Mars surface. The black dots along the axes are drawn every $0.1 \mathrm{R}_{\mathrm{M}}$ with the larger open squares drawn every $1 \mathrm{R}_{\mathrm{M}}$.

It is useful to explore the magnetic field magnitude along these field traces. Figure 3 is 
similar to Figure 2 but plots $|\mathrm{B}|$ instead of $|\mathrm{V}|$ on the selected field lines, with the same scale as that on the equatorial plane (i.e., saturated at $25 \mathrm{nT}$ ). Figure 3 shows that the very slow field line portions identified in Figure 2 are also regions of high (>25 nT) field strength. Of more interest is the "tips" of the "open" field lines; where the lines approach the inner boundary, the magnetic field strength plunges to zero. That is, there is very little magnetic field below the ionospheric density peak, which is good, but the magnetic field vectors in these last few grid cells still has some small magnitude. Moreover, the direction of this small vector is not horizontal but rather tipped enough to cause the trace to strike the inner boundary.

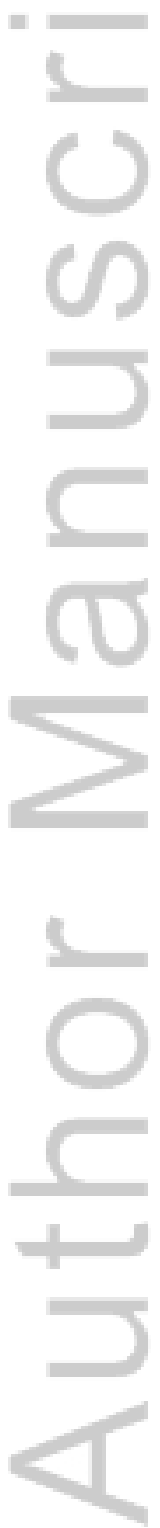


(a) Solar Max @ Perihelion

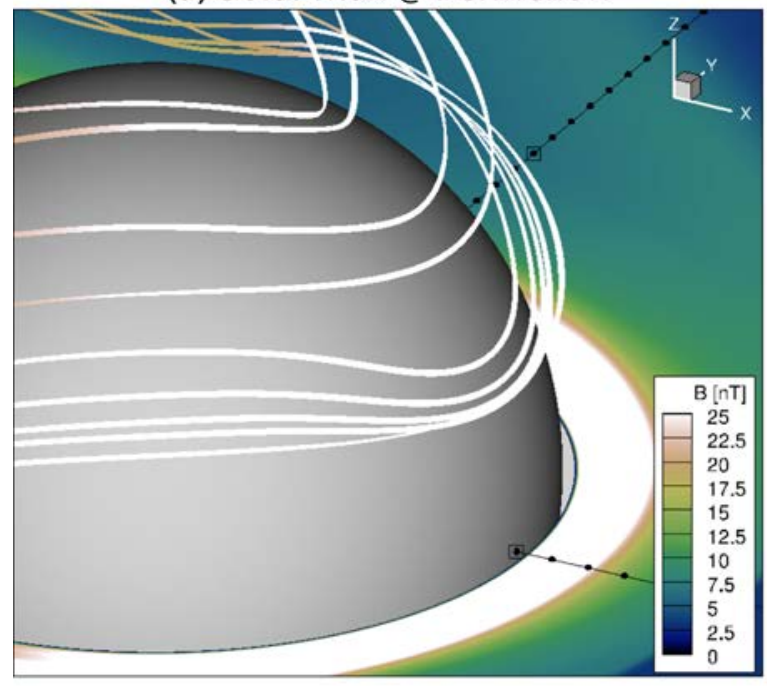

(c) Solar Min @ Perihelion

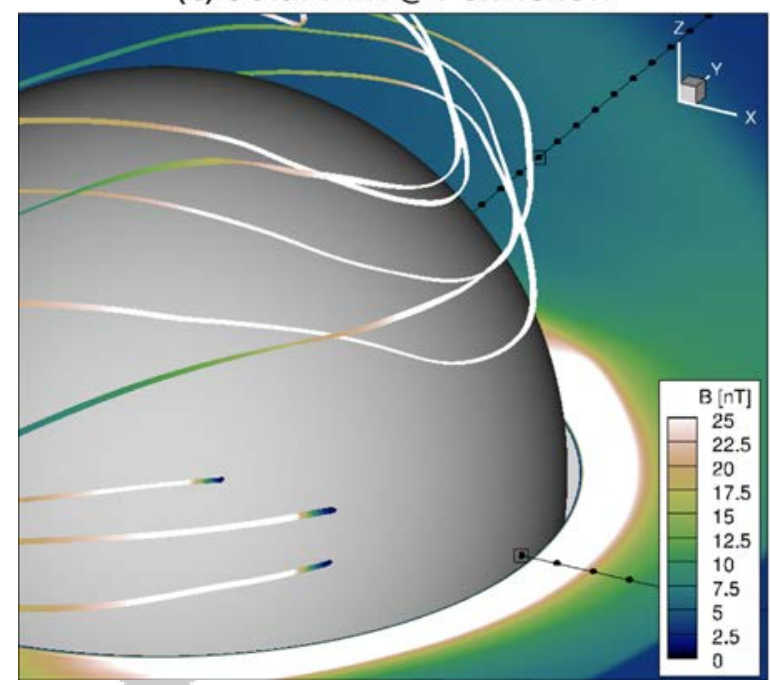

(b) Solar Max @ Aphelion

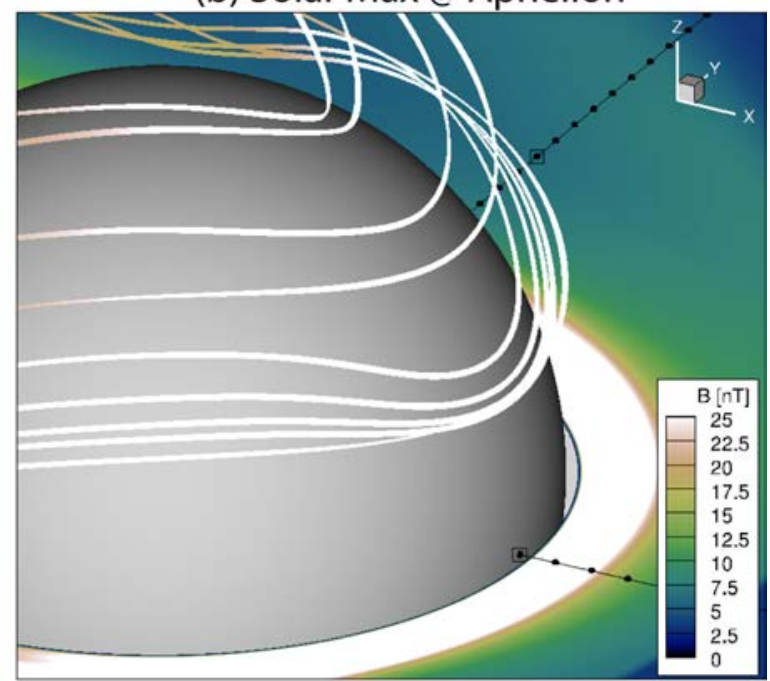

(d) Solar Min @ Aphelion

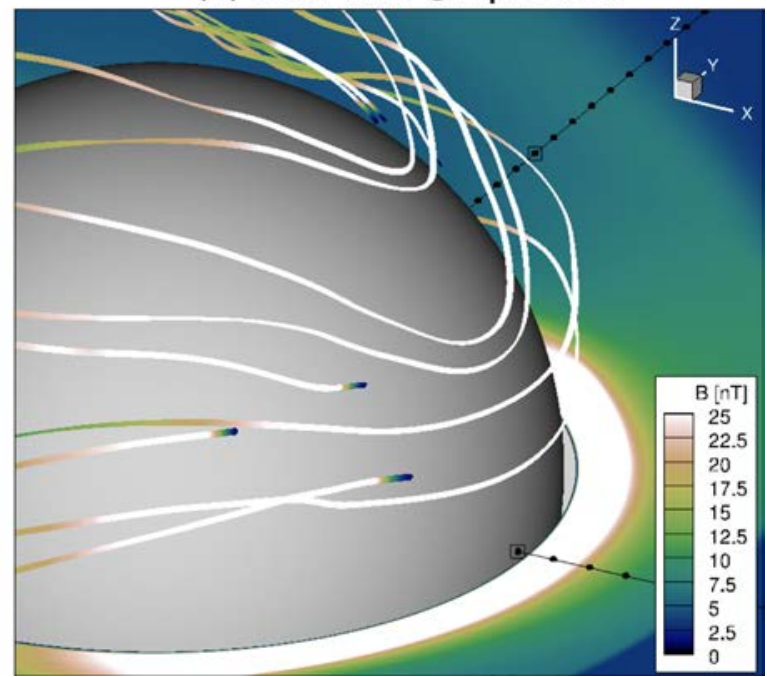

Figure 3. Same as Figure 2 except that the field lines are colored by the local magnetic field strength, with the same blue-green-pink color scale as the equatorial plane.

Taken together, Figures 1-3 demonstrate that there is not only a systematic difference in the Y location of the Mars tail current sheet between solar maximum and solar minimum 
conditions but also a difference in the distribution of magnetic flux within the dayside MPR between the two solar illumination cases.

\subsection{Testing the hypothesis}

Given this feature in the 3D plots, a hypothesis can be put forth that the draped IMF penetrates to lower altitudes at solar minimum than they do at solar maximum, and that this change in path through the Martian ionosphere influences the topology of the tail. To test this, an assessment of the minimum distance to Mars of the draped IMF lines can be conducted from the MHD results and the velocity of these field lines in this region.

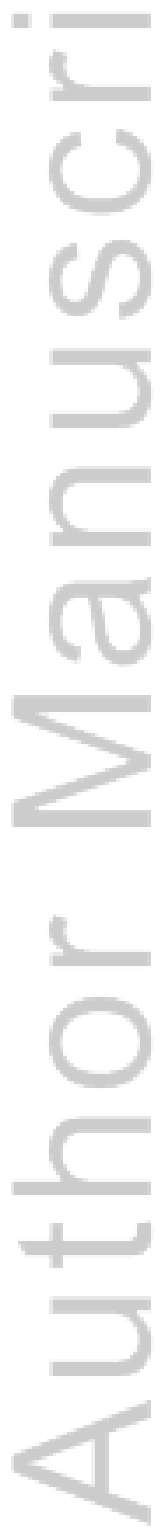


(a) Solar Max @ Perihelion

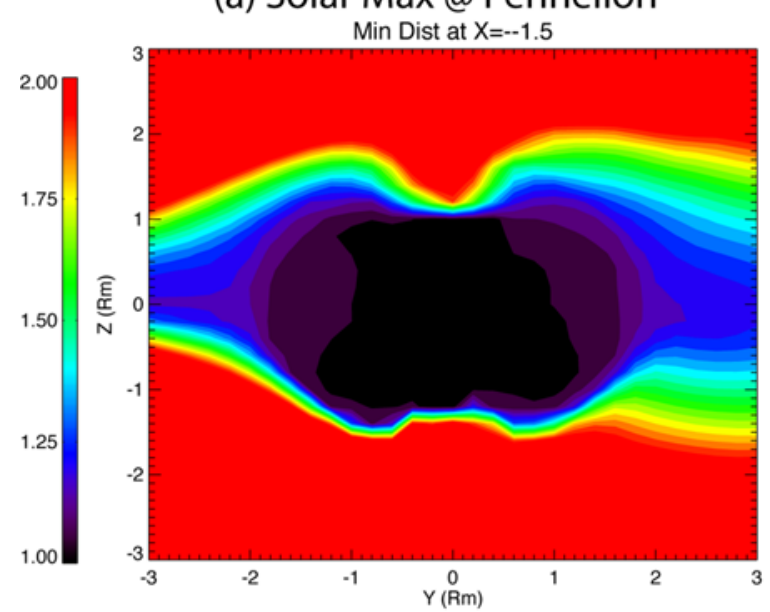

(c) Solar Min @ Perihelion

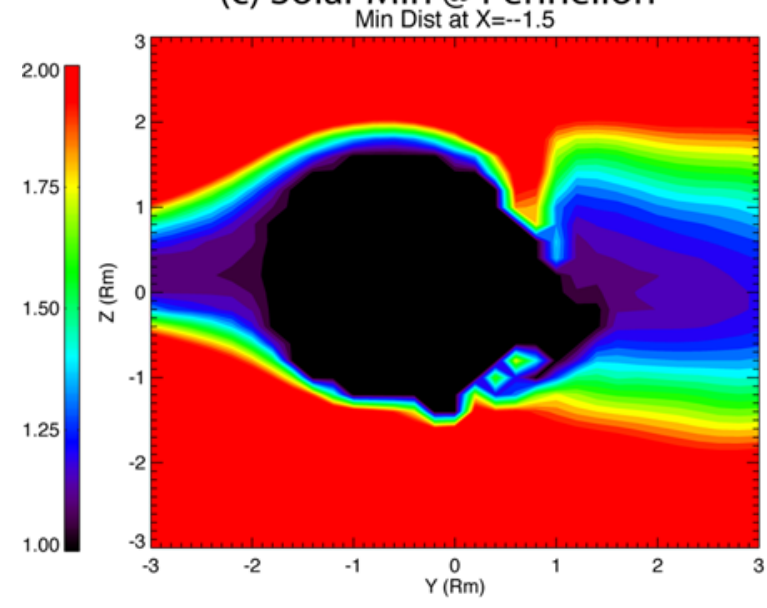

(b) Solar Max @ Aphelion

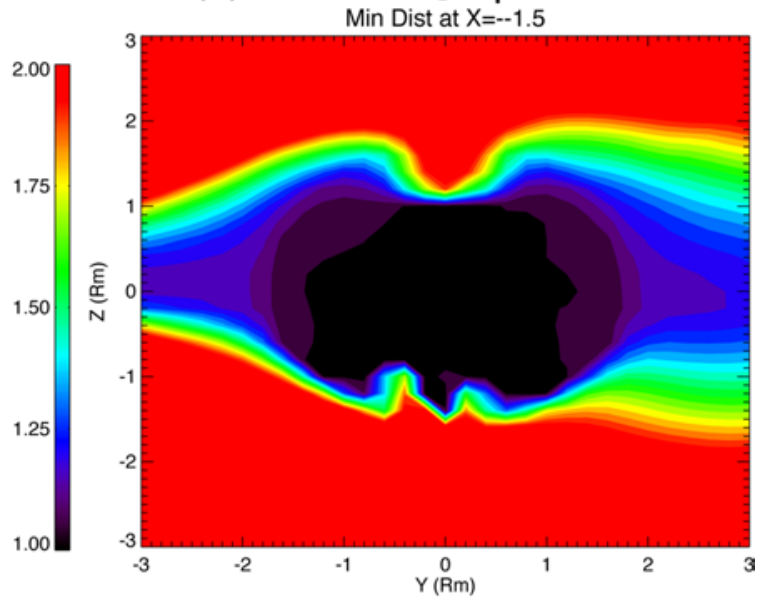

(d) Solar Min @ Aphelion

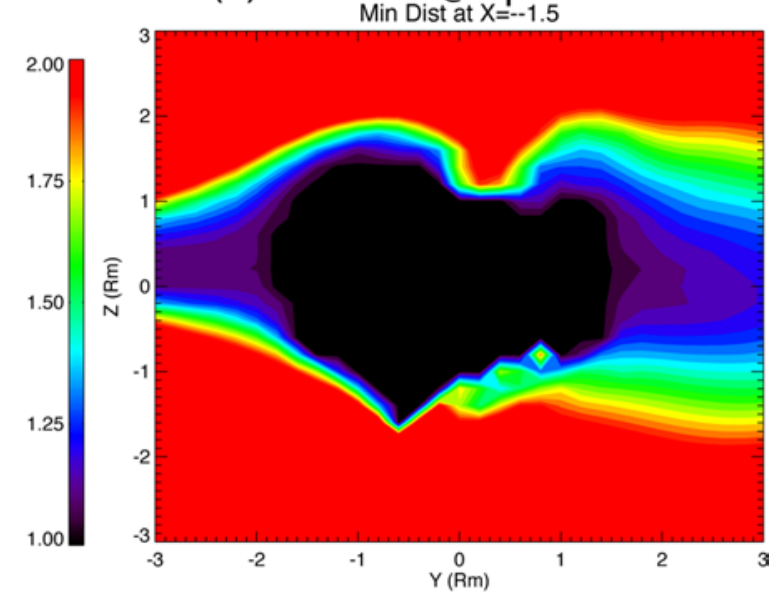

Figure 4. Minimum distance that a magnetic field line comes to Mars, based on field line traces through the MHD results starting at $-1.5 \mathrm{R}_{\mathrm{M}}$ downtail, for the 4 simulations in each panel, as labeled. Values are in Mars radii from the center of the planet and the range, from 1 to 2 , chosen to highlight near-Mars variations in closest approach.

Figure 4 shows such an analysis for the four simulations. Field line traces were started in a dense grid across the $X=-1.5 R_{M}$ plane, calculating the Mars-centric distance at each point along the trace. The closest approach value is shown in the plot, with the color range selected to highlight the field lines that come nearest to the planet. 
There is a black region in the center of each of the panels in Figure 4 for which the magnetic field line's closest approach to Mars is within $0.04 R_{M}$ from the surface. This is 135 $\mathrm{km}$, which means the field line dips below the density peak of the Mars ionosphere. Comparing the panels of Figure 4, this region increases as the solar EUV flux decreases, with the two solar minimum cases (lower row) having substantially larger black areas than the solar maximum cases (upper row).

Note that there is some amount of asymmetry and small-scale features in the panels of Figure 4. The structure close to the central black region is due to the 3D structure in the upper atmospheric densities included in the MHD simulations. Farther out, in the $\pm Y$ directions, the field within the magnetosheath reveals an asymmetry in the draping of the IMF. The strongly draped field lines on the $-\mathrm{Y}$ dawn side of the magnetosheath are focused closer to the equatorial plane than those on the $+\mathrm{Y}$ dusk side of the magnetosheath. That is, on the $-\mathrm{Y}$ dawn side, the draped field lines are more confined to the lobe, but this is not the case on the $+Y$ dusk side, where the draped field lines extend into the magnetosheath. This is true for both the solar maximum and solar minimum cases. Comparing solar maximum to solar minimum, the main difference is that the $-\mathrm{Y}$ dawn lobe region of very close approach to Mars substantially increases in size.

To take another step in the analysis, the velocity of each field line at its closest approach to Mars can be extracted from the simulation results. Figure 5 shows the absolute value of this quantity, on a logarithmic scale from $1 \mathrm{~m} / \mathrm{s}$ to $1000 \mathrm{~km} / \mathrm{s}$. The white region in the middle of each panel indicates values smaller than $1 \mathrm{~m} / \mathrm{s}$. These white regions largely match the black regions of Figure 4, increasing in size as the solar EUV flux decreases.

Figure 6 shows a very close-up view of magnetic field magnitude in the northern noon meridian. The same colorscale is used for $|\mathrm{B}|$ as in Figures 1-3 but the top of the scale has been increased to $53 \mathrm{nT}$ include nearly the full range of values (magnitudes above this threshold are colored white in the plot). Overlaid on the $|\mathrm{B}|$ color background are isocontours of $\log _{10}|\mathrm{~V}|$, showing contours every quarter decade from $-2(10 \mathrm{~m} / \mathrm{s})$ to $+2(100 \mathrm{~km} / \mathrm{s})$. The gray area on the right is Mars and the white stripe to the left of this is the gap region below $100 \mathrm{~km}$ altitude inside the inner boundary of the simulation domain.

First consider the magnetic field magnitudes in Figure 6. The two solar maximum runs (Figures $6 \mathrm{a}$ and $6 \mathrm{~b}$ ) do not saturate the chosen color scale, remaining just below the upper limit (see the peak values listed in Table 1). For the solar minimum runs (Figures 6c and 6d), there is a region where the magnetic field exceeds $53 \mathrm{nT}$ (although not by very much). Another feature to note is the pink-yellow color transition, which is $\sim 40 \mathrm{nT}$. For the solar maximum runs, this transition is at $600-700 \mathrm{~km}$ altitude. For the solar minimum runs, it is at $450-500 \mathrm{~km}$ altitude. Taken together, these key features of Figure 6 reveal that the pileup region for solar minimum is more compressed and closer to the planet than at solar maximum. 
Figure 6 reveals the drift speeds in the regions of highest magnetic field. The "1" isocontour (i.e., $10 \mathrm{~km} / \mathrm{s}$, or 5.7 minutes per $\mathrm{R}_{\mathrm{M}}$ ) is very close to the $\sim 40 \mathrm{nT}$ transition just discussed for all four cases. This means that the most of the magnetic pileup region is moving rather slowly, taking many minutes (if not hours) slip around Mars, even for solar maximum. The magnetic field peak is not associated with a particular speed but rather there is a gradient across it in all of the plots, with speeds dropping all the way to the "-2" isocontour $(10 \mathrm{~m} / \mathrm{s})$ within the peak $|\mathrm{B}|$ portion. For those field lines that eventually thread below the peak and intersect the inner boundary, the velocity on that field line reaches the imposed inner boundary velocity of zero, thus accounting for the very low velocities seen in Figure 5.

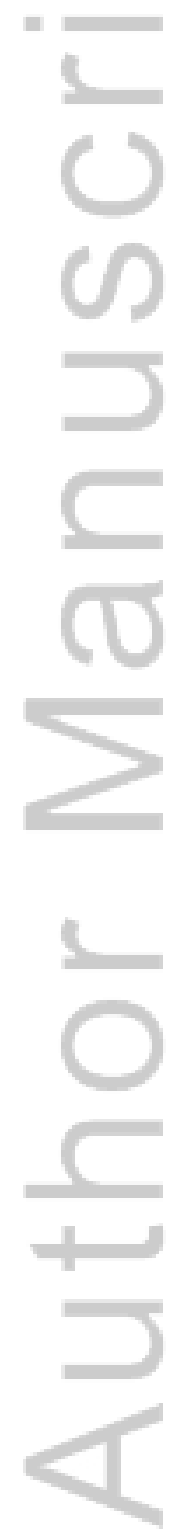

This article is protected by copyright. All rights reserved. 
(a) SolarMax @ Perihelion

log10(IVI) @ min Alt; X=--1.5

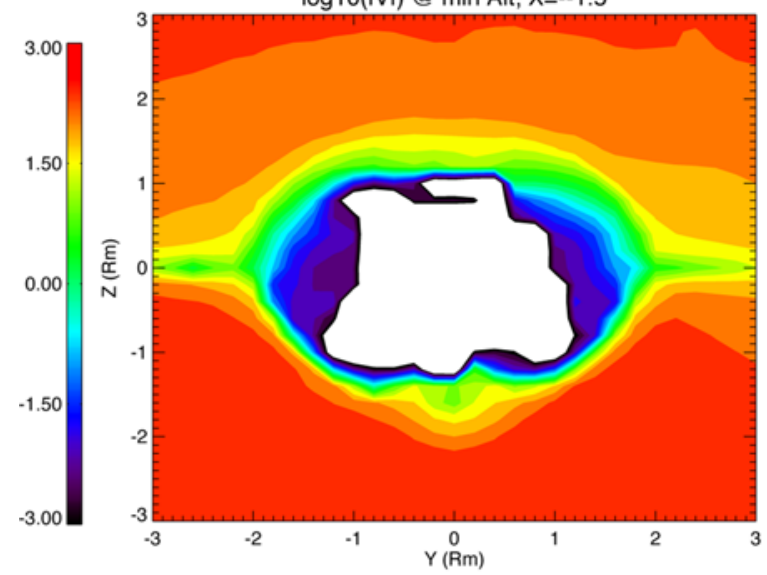

(c) Solar Min @ Perihelion log10(IVI) @ min Alt; $X=--1.5$

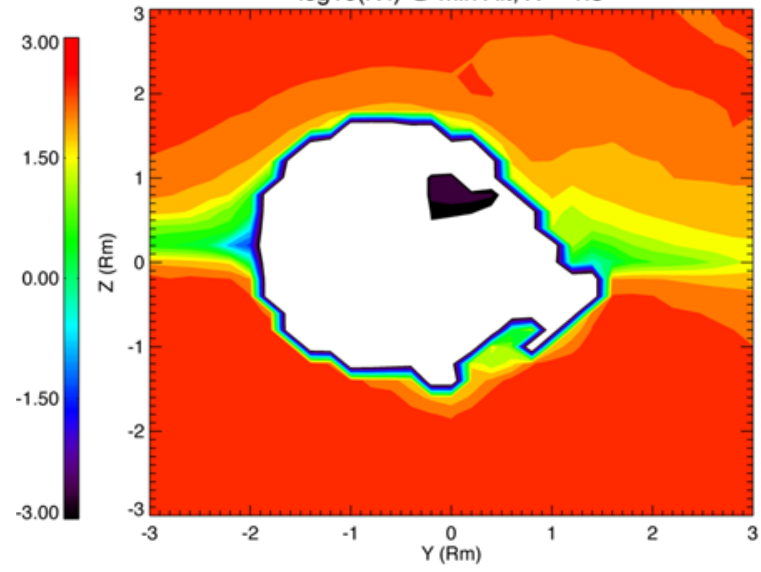

(b) Solar Max @ Aphelion

$\log 10(\mathrm{IVI}) @ \min$ Alt; $\mathrm{X}=--1.5$

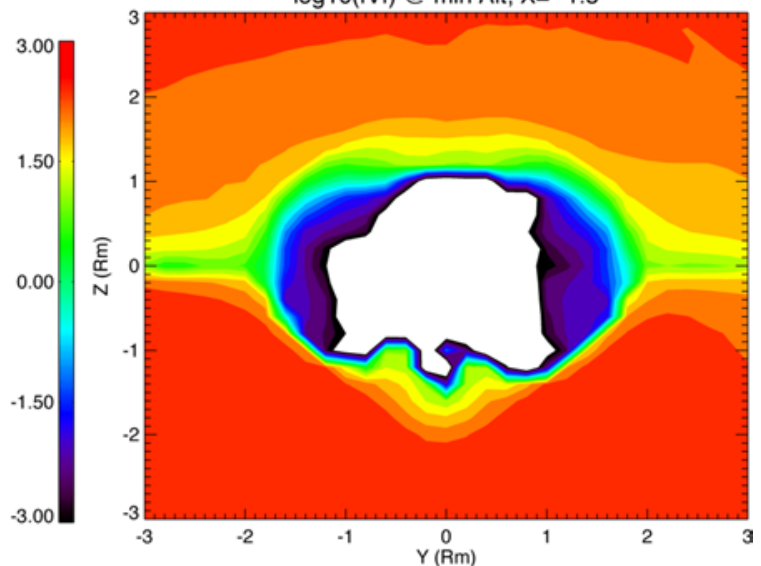

(d) Solar Min @ Aphelion $\log 10(\mathrm{IVI}) @$ min Alt; $X=--1.5$

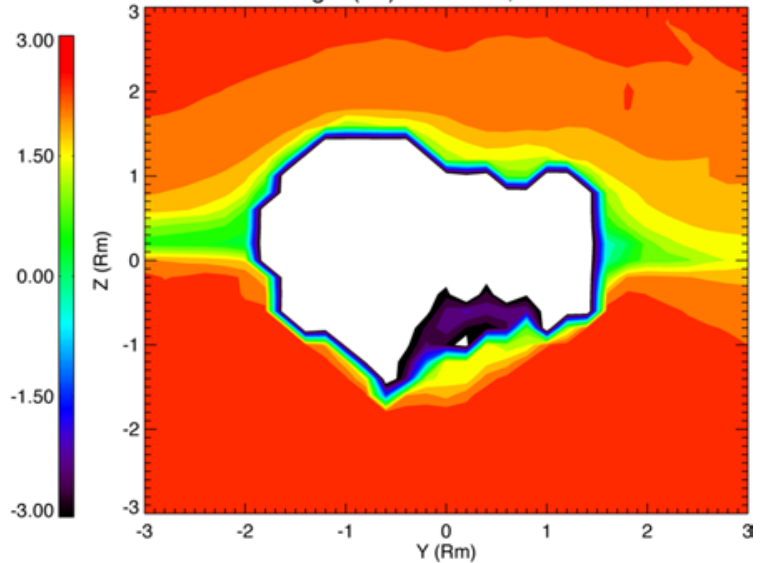

Figure 5. $\log _{10}$ of the magnitude of velocity, in $\mathrm{km} / \mathrm{s}$, at the closest approach location of each magnetic field trace, which were started at X-1.5 $\mathrm{R}_{\mathrm{M}}$ downtail, for the 4 simulations in each panel, as labeled. White regions are values below the lowest value on the color scale.

Ionospheric density plays a key role in this process. Figure 7, seen from the same viewing angle and in the same format as Figure 6, shows total ion mass density $\left(\mathrm{amu} / \mathrm{cm}^{3}\right)$, green-blue-lavender color bar on a logarithmic scale) with the X-Z component of the velocity vector overlaid as arrows. The vectors are drawn every grid cell in this plane, so the numerical grid is revealed, except at low altitudes where the vectors become too small to see (the grid here 
is dense, with $5 \mathrm{~km}$ radial spacing). The vector length is scaled so that a vector reaching horizontally between two polar angle lines of cells is $70 \mathrm{~km} / \mathrm{s}$.

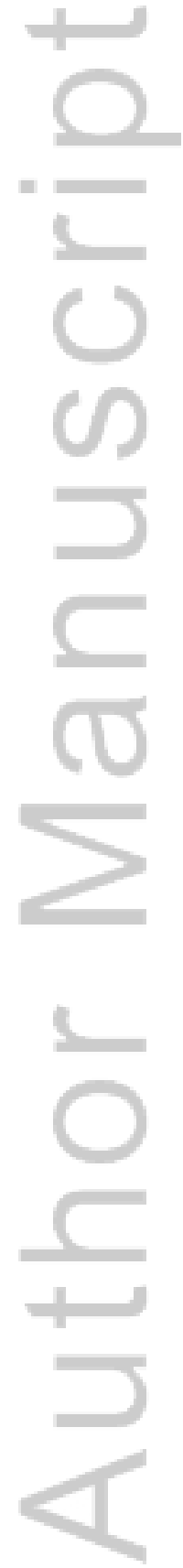

This article is protected by copyright. All rights reserved. 
(a) Solar Max @ Perihelion

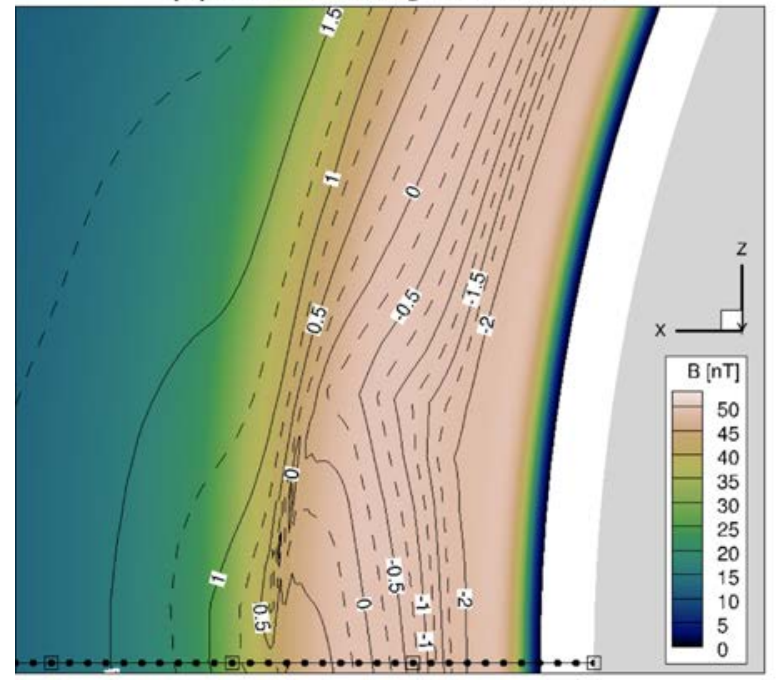

(c) Solar Min @ Perihelion

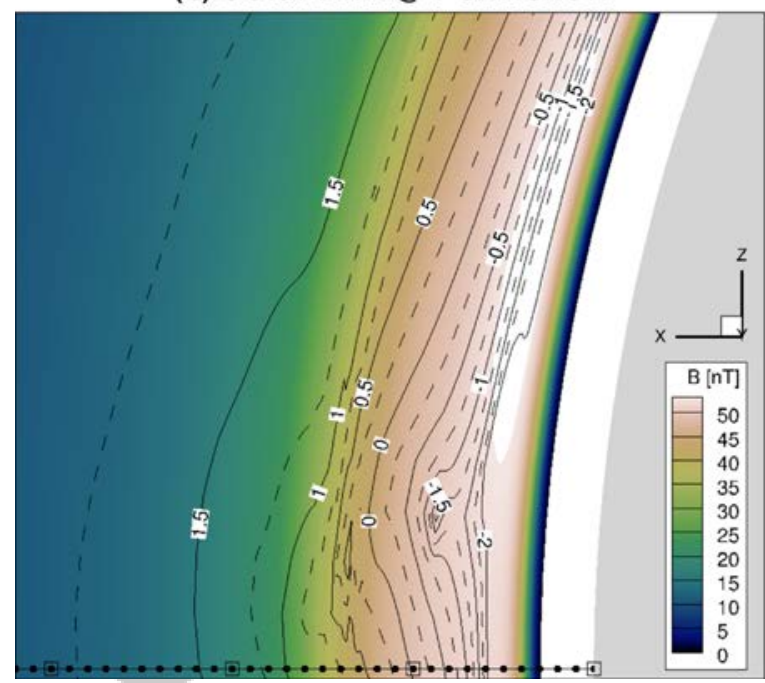

(b) Solar Max @ Aphelion

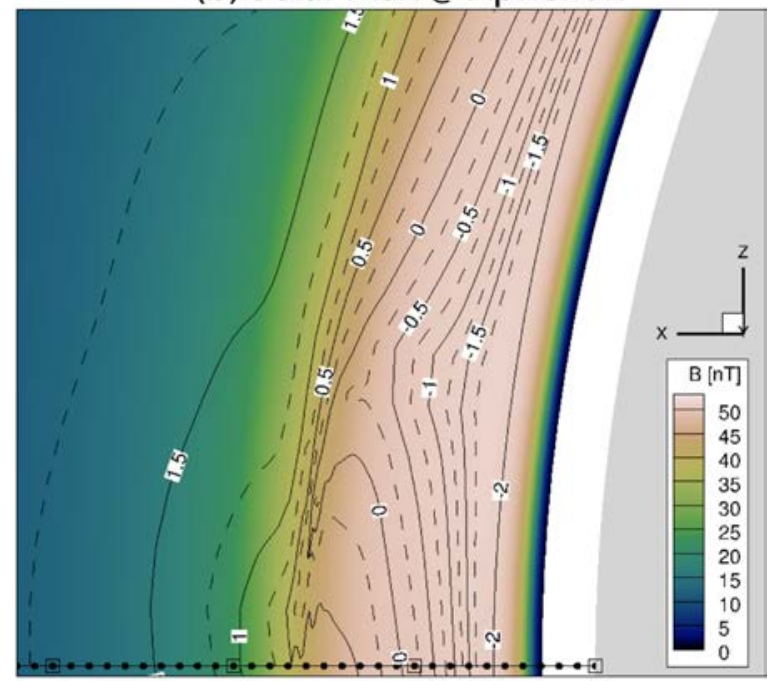

(d) Solar Min @ Aphelion

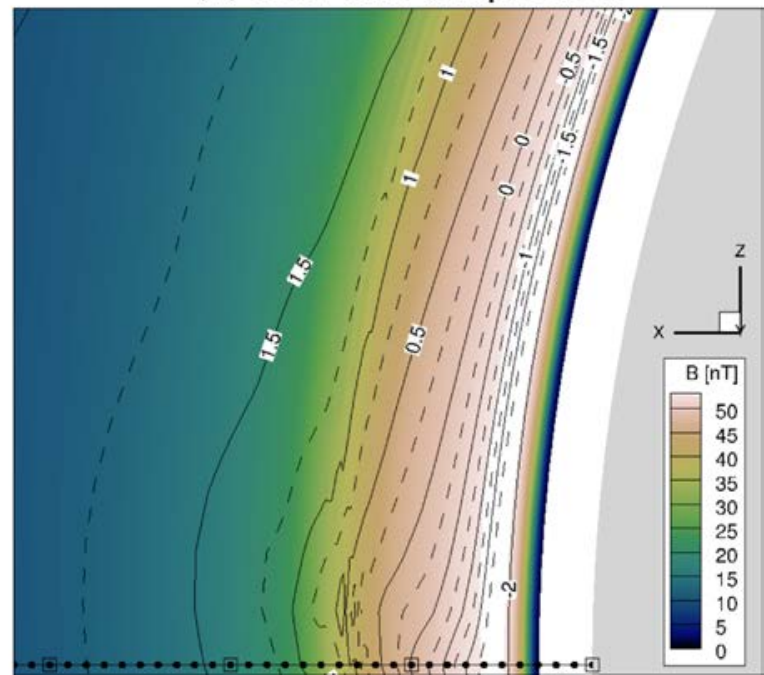

Figure 6. Closeup view of the noon meridian, as seen from dusk. Shown in color is the magnitude of the magnetic field (blue-green-pink color scale, note the peak value is different from earlier plots) and overlaid are contours of the $\log _{10}$ of speed (magnitude of velocity, with contours every quarter decade from -2 (i.e., $10 \mathrm{~m} / \mathrm{s}$ ) to 2 (i.e., $100 \mathrm{~km} / \mathrm{s}$ ). The black dots along the axes demark $0.01 \mathrm{R}_{\mathrm{M}}$ spacing (i.e., $34 \mathrm{~km}$ ), with larger open squares drawn every $0.1 \mathrm{R}_{\mathrm{M}}$ along the axes.

Figure 7 shows that the ionosphere is more extended at solar maximum than at solar 
minimum. The region of relatively high mass density, the lavender-blue transition $(\sim 105$ $\mathrm{amu} / \mathrm{cm}^{3}$ ), is in the $350-400 \mathrm{~km}$ altitude range at solar maximum and in the $250-300 \mathrm{~km}$ altitude range at solar minimum. Comparing Figure 7 with Figure 6, it can be seen that the peak $|\mathrm{B}|$ portion of the MPR is within this high ion mass density layer. The draped IMF penetrates into the dayside ionosphere and then progresses rather slowly to the polar region where it can move upward and be released from this mass loading. Another feature to be seen in this comparison is that the blue-green color transition in the mass densities $\left(\sim 100 \mathrm{amu} / \mathrm{cm}^{3}\right)$ is very closely aligned with the $\sim 40 \mathrm{nT}$ transition in the magnetic field.

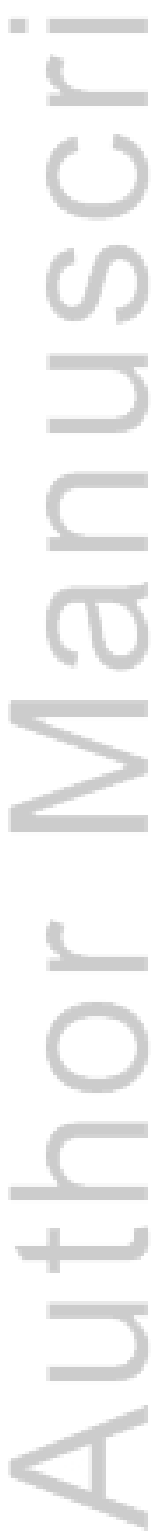


(a) Solar Max @ Perihelion

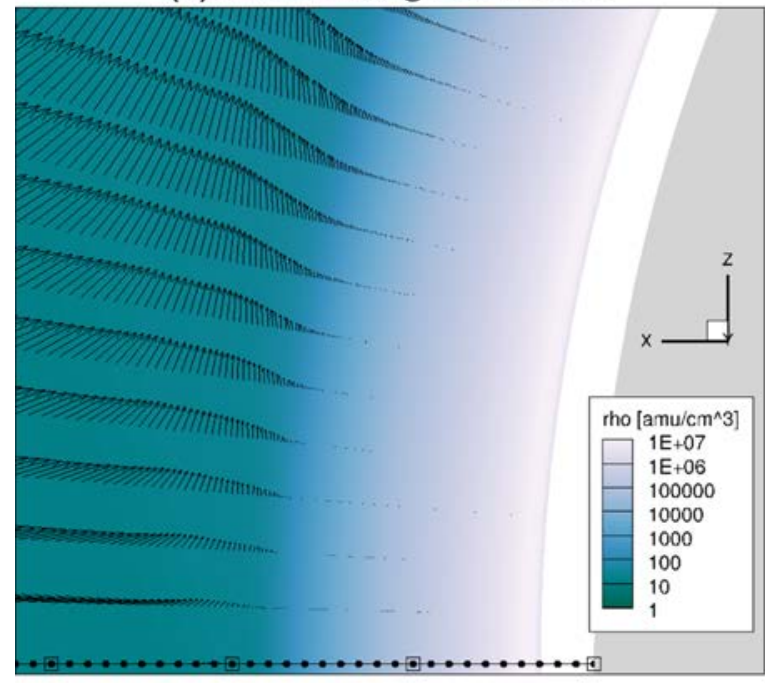

(c) Solar Min @ Perihelion

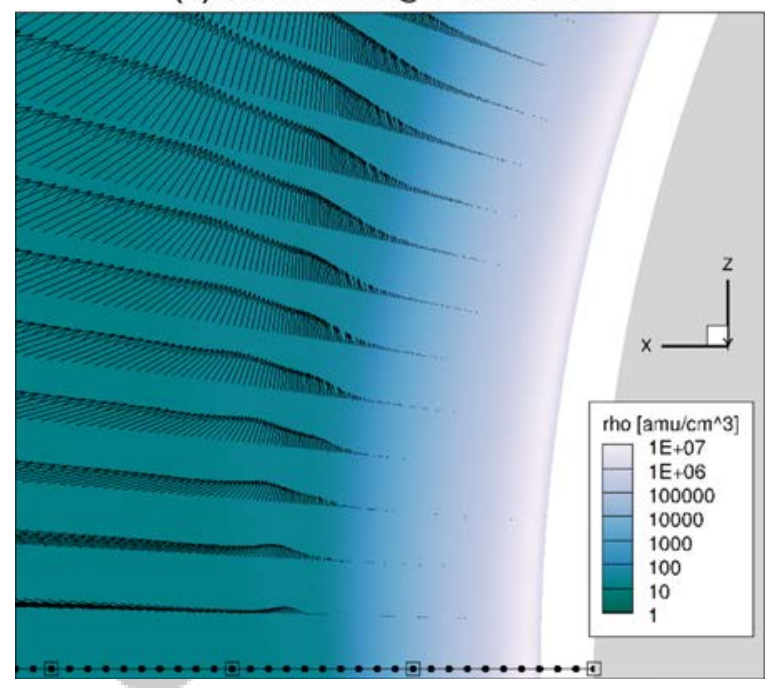

(b) Solar Max @ Aphelion

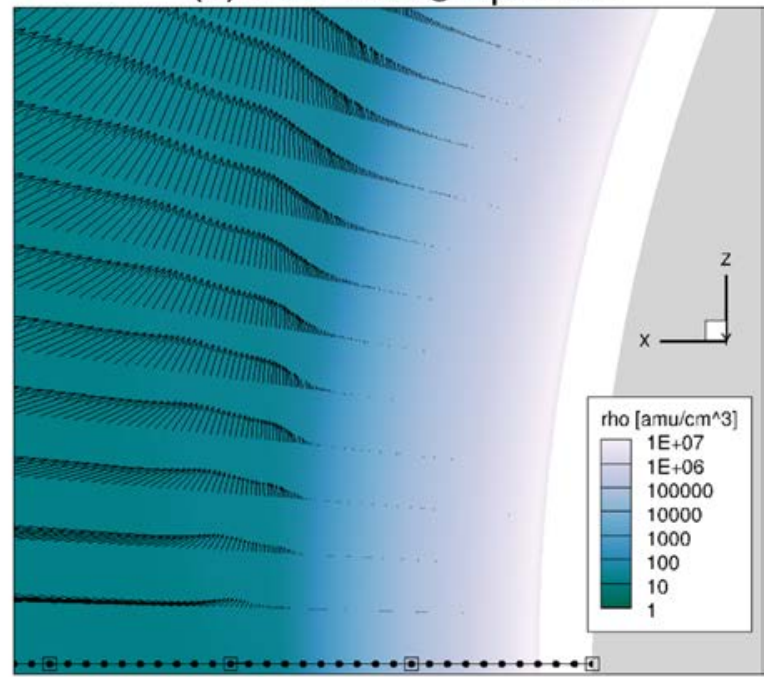

(d) Solar Min @ Aphelion

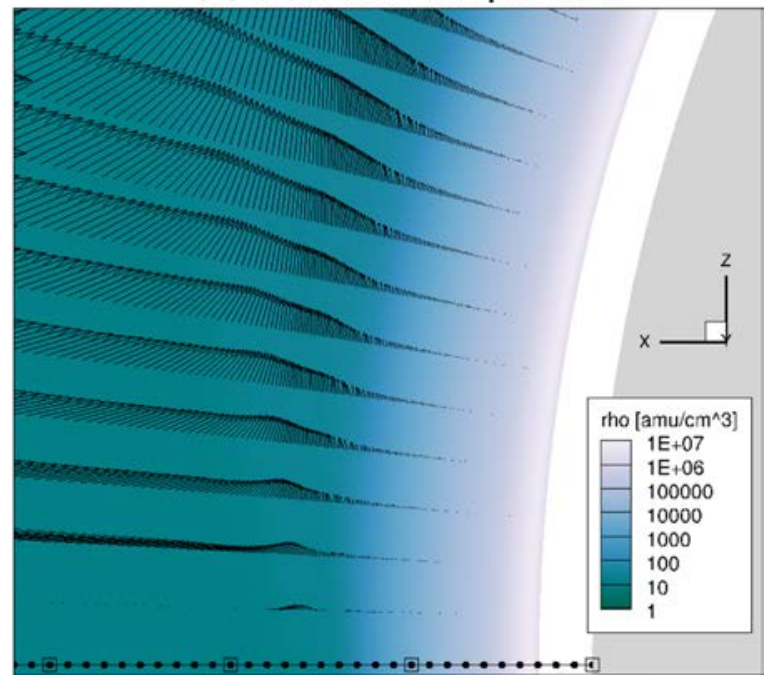

Figure 7. Same view and format as Figure 6 except that the color quantity is total ion mass density $\left(\mathrm{amu} / \mathrm{cm}^{3}\right)$ on a logarithmic scale (green-blue-lavender color bar) and overlaid are velocity vectors showing the $\mathrm{X}-\mathrm{Z}$ component in this plane. The vectors are scaled such that a horizontal vector spanning exactly the spacing between two polar angle lines of grid cells is $70 \mathrm{~km} / \mathrm{s}$.

To be even more quantitative, Figure 8 shows the pressure along the subsolar line from 
the four simulation results. There should be a rough pressure balance along this line to hold off the incoming solar wind plasma dynamic pressure (green dashed line). The solar wind thermal plasma pressure (magenta dotted line) is the dominant term in the sheath region, and closer to the planet this term is replaced by magnetic pressure (red dashed line) in the MPR. Very close to the inner boundary, the ionospheric thermal plasma pressure becomes a significant term. The ionospheric thermal plasma curve (yellow dotted line, essentially the same as the blue thin solid line between 1.0 and $1.2 \mathrm{R}_{\mathrm{M}}$ ) shows a clear difference between the solar maximum cases and the solar minimum cases, with the solar max ionospheres reaching to higher altitude, as seen in Figure 7. The plots in Figure 8 also show that the peak magnetic field value in or near the ionosphere is larger for solar minimum than for solar maximum, as seen in Figure 6.

A final illustration of the magnetic connection to the inner boundary is shown in Figure 9. These plots show a view from local midnight, looking back toward the Sun, so the $+Y$ (duskward) direction is to the left. Magnetic field magnitude is shown in the $\mathrm{X}=0$ plane with the same colorbar as before but with a different topmost value to highlight the magnetic features in this plane. The sphere is no longer at the Mars surface but rather at $120 \mathrm{~km}$ altitude, below the nightside ionospheric peak but above the inner boundary of the simulation domain. On this sphere is shown the radial component of the local magnetic field, $\mathrm{Br}$, with an orange-whitepurple color scale. Also drawn on the sphere are isocontours of $\mathrm{Br}$. The dots along the axes indicate steps of $0.1 \mathrm{R}_{\mathrm{M}}$.

Figure 9 shows that the radial fields are larger in the solar minimum cases than the solar maximum cases. To be more quantitative, the integral of $|\mathrm{Br}|$ on the $120 \mathrm{~km}$ altitude surface for each case is listed in the eighth column of Table 1. The sign of $\mathrm{Br}$ was removed before the integration so this is a value of all "open magnetic flux" in each case. Separating the integral by $\mathrm{Br}$ sign, the downward value is slightly larger than the upward value in all four cases, but they are close. The peak downward and upward $\mathrm{Br}$ values at $120 \mathrm{~km}$ altitude are listed in columns 9 and 10, respectively. All three of these columns yield the same result that there is more radial magnetic flux below the ionosphere in the solar minimum cases than in the solar maximum cases.

The currents and $\mathrm{JxB}$ forces in the magnetotail were also examined for this study. Because it is an away-sector IMF, the current systems are as expected for this configuration, with a central tail current flowing in the $+Z$ direction, a counterclockwise current (as viewed from the Sun) around the dawnside lobe and the clockwise current around the duskside lobe. The peak value of the central current sheet density is on the order of $5-10 \mathrm{nA} / \mathrm{m}^{2}$ and the peak current densities in the outer cylinders of current around the lobes are between 0.1 and $1 \mathrm{nA} / \mathrm{m}^{2}$. The associated $\mathrm{JXB}$ forces follow expectations, with $\mathrm{a}-\mathrm{X}$ oriented force in the central tail and "outward" forces near the outer boundary of each tail lobe. As listed in Table 1, the magnetic field in the current sheet is less than $1 \mathrm{nT}$, much less than the 10-20 nT peak values in the lobes, 
and the sharp kink in the magnetic field lines confirms that this is essentially a tangential discontinuity.

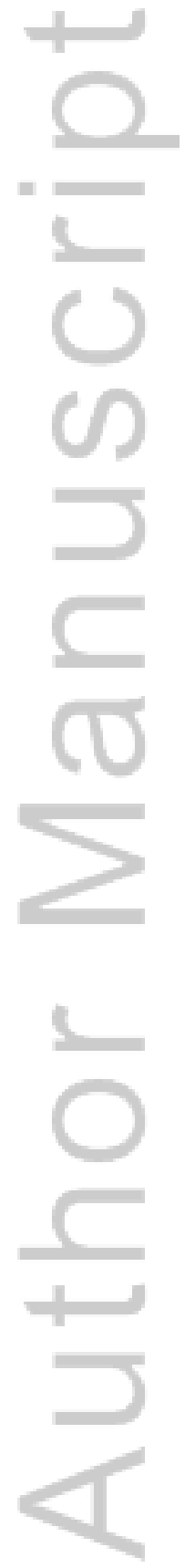


(a) SolarMax@ Perihelion

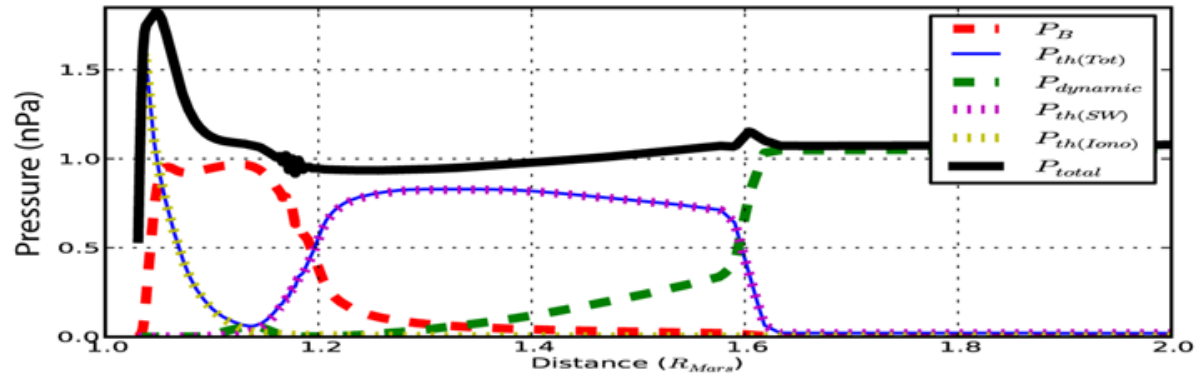

(b) SolarMax @ Aphelion

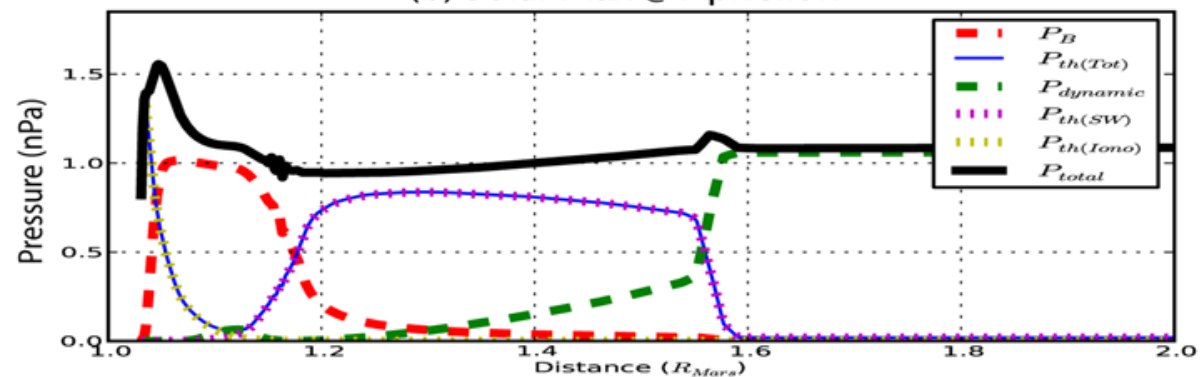

(c) Solar Min @ Perihelion

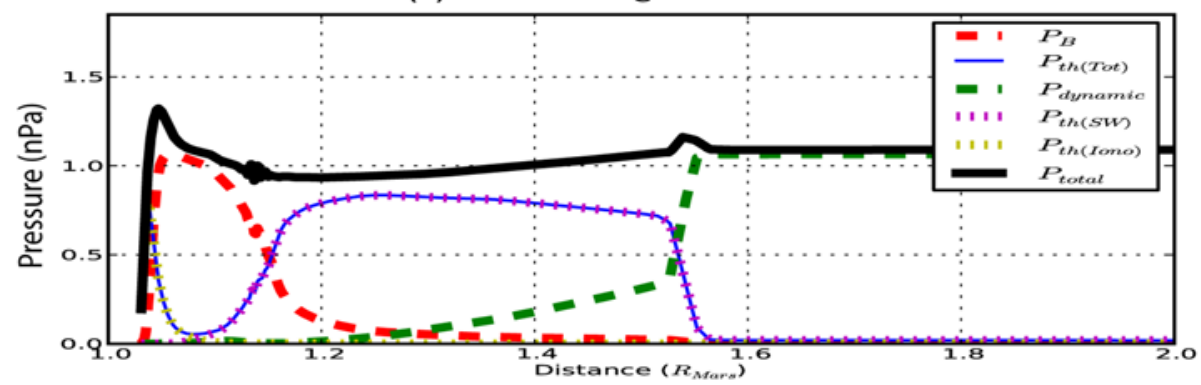

(d) Solar Min@ @ Aphelion

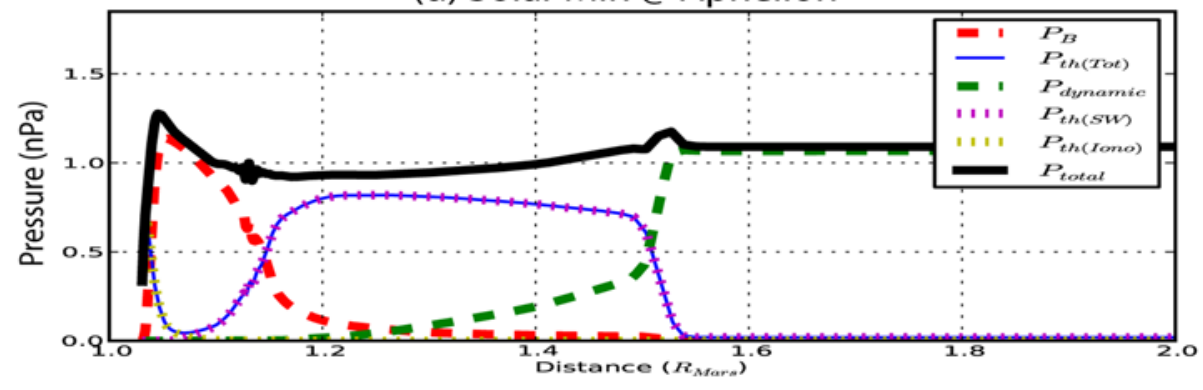

Figure 8. Line plots of pressure (in $\mathrm{nPa}$ ) along the $+\mathrm{X}$ axis (subsolar line) for the four simulations, showing the total pressure (black solid) and its components: magnetic pressure (red dashed), thermal plasma pressure from all species (blue solid), dynamic plasma pressure (green dashed), thermal plasma pressure from solar wind protons (magenta dotted), thermal plasma pressure from ionospheric species (yellow dotted).

\section{Discussion}

This article is protected by copyright. All rights reserved. 
The Mars magnetotail current sheet is located on the $-Y$ side of the noon-midnight

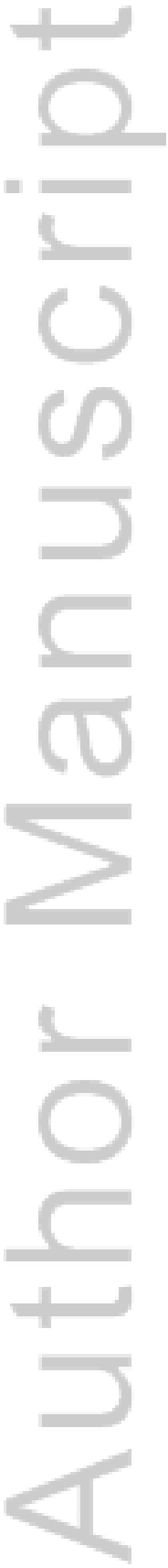

This article is protected by copyright. All rights reserved. 
meridian for the solar maximum cases and then moves to the $+Y$ side of the $-\mathrm{X}$ axis for the solar

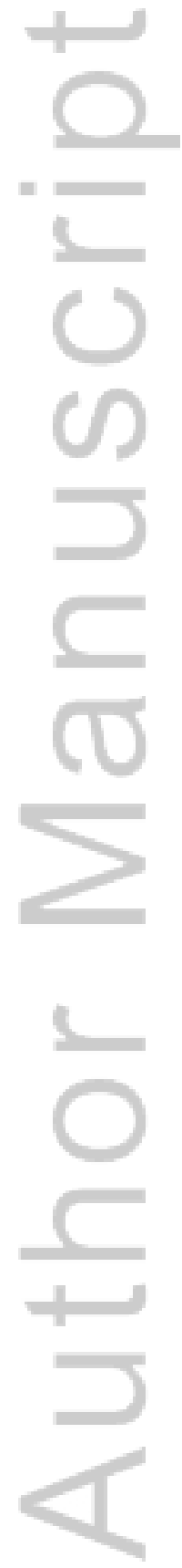

This article is protected by copyright. All rights reserved. 
(a) Solar Max @ Perihelion

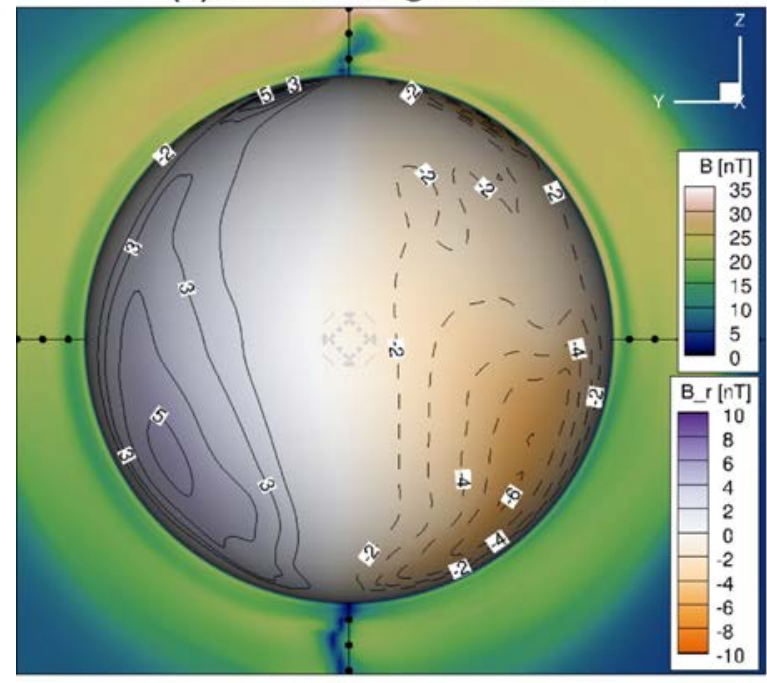

(c) Solar Min @ Perihelion

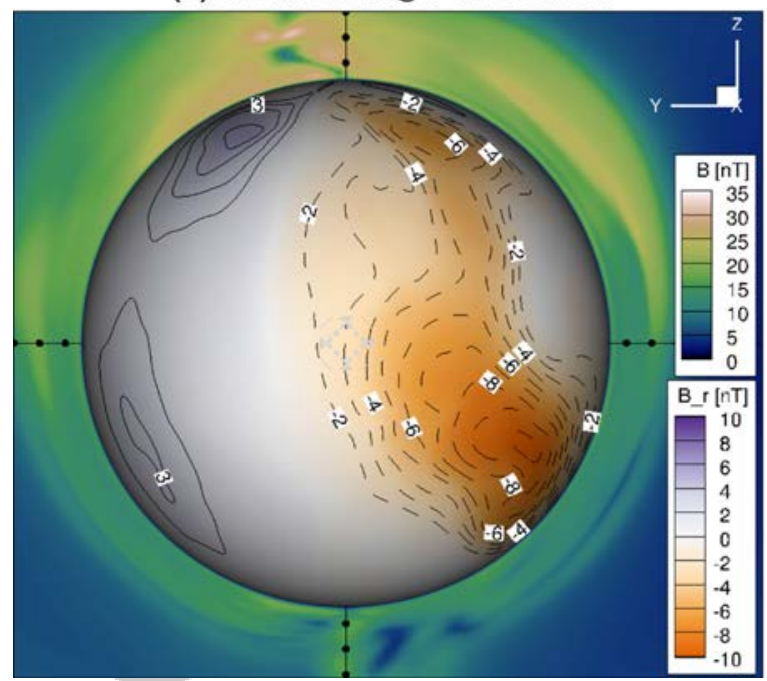

(b) Solar Max @ Aphelion

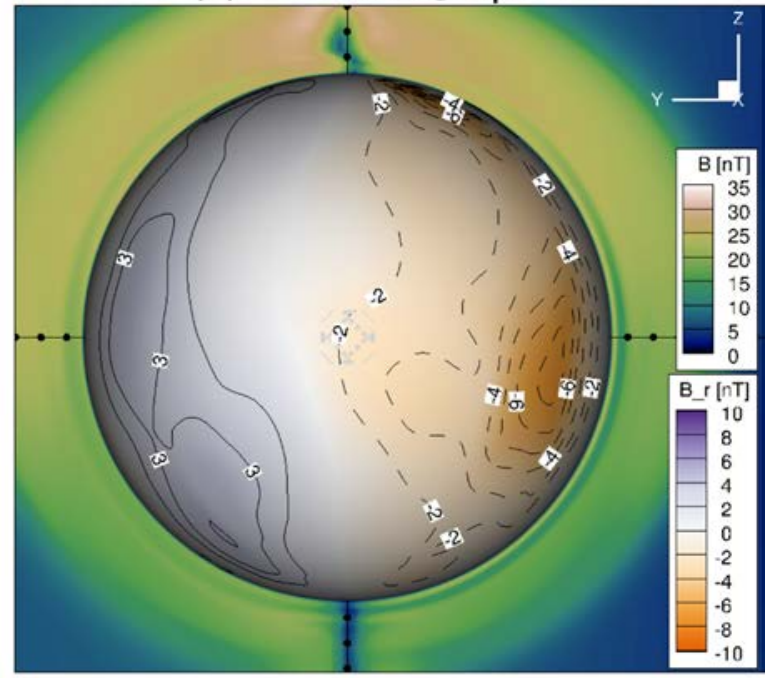

(d) Solar Min @ Aphelion

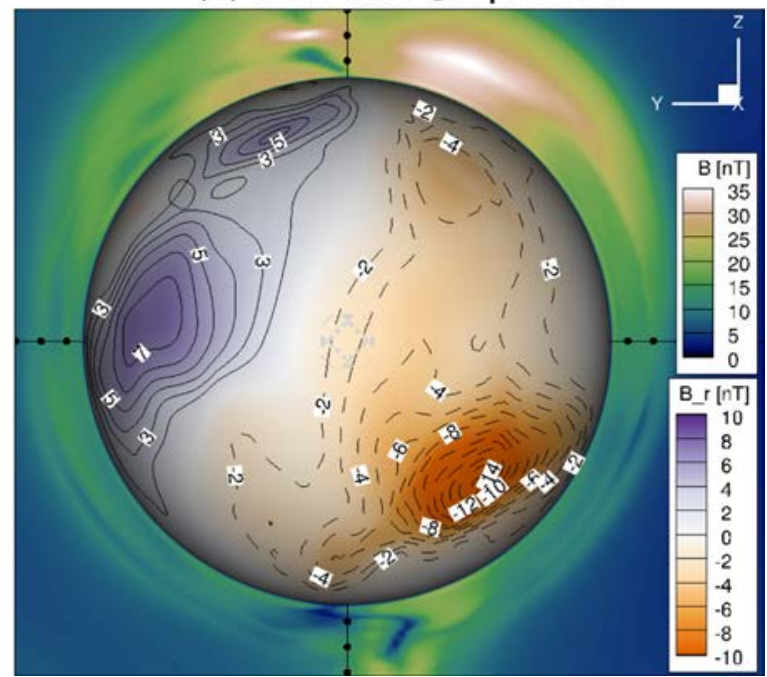

Figure 9. View from the $-X$ axis directly towards the Sun. Magnetic field intensity is shown in the $X=0$ plane (blue-green-pink color scale, note that it is a different peak value from earlier plots) and the sphere at $120 \mathrm{~km}$ altitude is colored with the radial component of the magnetic field (purple-white-orange scale). Contours of the $\mathrm{Br}$ value are also drawn on the sphere, with positive (outward) values as solid lines and negative (inward) values as dashed lines. Note that the $-1,0$, and $1 \mathrm{nT}$ lines are omitted.

minimum cases. The results show that the $40 \%$ EUV seasonal change from perihelion to 
aphelion shifts the tail current sheet a little bit, but only the $290 \%$ solar cycle change from maximum to minimum is sufficient to move the tail current sheet from a-Y location over to the $+Y$ side. That is, for the solar maximum conditions, the Mars magnetotail is Venus-like, with a systematic dawn shift to the location of the current sheet. For solar minimum conditions, the Mars magnetotail is different.

The only difference between the runs are the atmospheric densities and solar flux input values. These are otherwise identical simulation configurations with symmetric initial and boundary conditions. The primary asymmetry about the $\mathrm{Y}=0$ plane is the Parker spiral angle IMF, which creates an S-shaped magnetic topology. The 3D neutral atmosphere values also create a small bit of asymmetry in the MHD solution (as shown in Dong et al. [2015a]).

To understand the physical process causing this shift in the asymmetry of the tail, it is useful to remember that the Mars ionosphere is, by itself, too weak in thermal plasma pressure to stand off the solar wind dynamic pressure [see, e.g., the discussion of this in Nagy et al., 2004]. Magnetoplasma pressure balance along the subsolar line, however, causes a stagnation and accumulation of the IMF into a MPR. The inner edge of this MPR is often within the ionosphere, magnetizing the top portion of the planets upper atmosphere. This slows the progression of the field line slippage past the planet, as seen in the weathervaning magnetic topology close to the planet in the northern hemisphere in Figures 1-3 [cf., Brain et al., 2006].

The extent of this ionospheric magnetization changes with ionospheric thermal pressure magnitude, a quantity controlled by the solar EUV intensity. At solar maximum, the ionosphere is relatively strong and the stagnated IMF in the MPR slips past Mars with relative ease. At solar minimum, however, a higher fraction of the draped IMF field lines are pushed quite deeply into the ionosphere, making slippage past the planet more difficult. This deeper penetration of the IMF seen by the increase the larger "black" regions in Figure 4 between the upper and lower rows, and the slowing down of the slippage rate is seen by the increased "white" region in Figure 5, again comparing the upper and lower rows. In the solar minimum at aphelion case, a few of the field lines reach the bottom of the ionosphere, as evidenced by the field traces touching the inner boundary of the simulation domain in Figures 1-3 and the $\mathrm{Br}$ values in Figure 8.

The model does not resolve the magnetic topology below the innermost shell of true grid cells. The code can, however, account for nonhorizontal magnetic field vectors in that first cell above the inner boundary. Nearly any vertical tilt to the magnetic field in a cell within this first shell will yield a magnetic field trace that "touches" the inner boundary. The horizontal velocity in these cells will eventually move the magnetic flux towards the nightside, where forces will cause it to rise away from the planet and head downtail. The velocities in the cells of this first shell, however, are usually quite small, preventing rapid transfer of magnetic flux at these altitudes. The fact that any magnetic field line traces touch the inner boundary and become open flux is, of course, a numerical artifact. In reality, these field lines are draped just like those at slightly higher altitudes. The fact that some magnetic flux touches the inner boundary does not change the physical finding that, at solar minimum, the MPR is closer to the planet with a larger 
magnetic field in the ionosphere where the flow speeds are tiny relative to the solar wind speed, and that this change on the dayside influences the magnetic topology of the tail and the $Y$ location of the current sheet.

This slippage rate changes the magnetic field in the lobes. As seen in Figure 1, a Parker spiral magnetic field configuration means that the field line crosses the bow shock at different $\mathrm{X}$ locations on the dawn and dusk side of the planet. Specifically, it crosses farther downtail on the $+\mathrm{Y}$ dusk side than on the $-\mathrm{Y}$ dawn side, regardless of the toward-or-away direction of the IMF. The flow in the magnetosheath is slowed as the shocked solar wind is redirected around Mars, so these field lines bend back sunward inside of the bow shock. For the Venus case and for Mars at solar maximum, the draped IMF lines slip by the planet relatively quickly, so there is only a modest buildup of magnetic pressure from this sunward excursion of the lines. In addition, the field line is only modestly bent back sunward. For the case of Mars at solar minimum, more of the draped IMF moves slower through the ionosphere, increasing the slippage time and therefore elongating the draped IMF lines within the magnetosheath and MPR. This elongation also aligns the field lines in a more sunward-antisunward direction rather than a Parker spiral direction. Furthermore, the nonlaminar structure of the magnetosheath, where kinetic processes and nonlinear effects are prevalent, could be playing a significant role in altering the slippage rate and creating this dawn-dusk shift in the Mars tail current sheet.

This increase in sunward-aligned lobe magnetic field is seen in Figure 1, comparing the top and bottom rows. The increased slippage time means that a larger amount of magnetic flux must come back from the tail all the way to the dayside of the planet, not just partly back (which would be the case if it slipped quickly). On the $-Y$ dawn side of the tail, this magnetic flux comes back through the lobe. On the $+Y$ dusk side, this magnetic flux is not confined to the lobe but can extend into the magnetosheath. This flaring of the IMF into the magnetosheath somewhat reduces the magnetic flux in the $+Y$ duskside lobe, even at solar maximum. Remember, though, that the Parker spiral orientation of the IMF sets up a $\pm Y$ asymmetry in the amount of magnetic field inside the bow shock, favoring the $+Y$ dusk side of the magnetotail. For the Mars solar maximum case, there is still more magnetic flux in the $+Y$ duskside lobe, even with this flaring of the draped IMF into the $+\mathrm{Y}$ duskside magnetosheath. For the Mars solar minimum case, the increased magnetic flux in the $-Y$ dawnside lobe is finally larger than the $+Y$ duskside lobe, and the Mars magnetotail current sheet shifts to the $+Y$ duskside of the midnight meridian.

The very slow slippage rates found in this study imply that the tail should stretch many Mars radii behind the planet. Xu et al. [2016] analyzed this in MHD results and found that the unity isocontour for plasma beta (one measure of the tail lobe outer boundary) extends 15-20 $R_{M}$ downtail. Observational evidence exists for a clear two-lobe structure at $\mathrm{X} \sim-2.9 \mathrm{R}_{\mathrm{M}}$ downtail from the Phobos 2 spacecraft [Yeroshenko et al., 1990]. Follow-on studies with this data set confirmed the dominance of an induced magnetotail structure at this distance [e.g., Riedler et al., 1991; Schingenschuh et al., 1992]. Unfortunately, no long-duration magnetic field observations 
exist at farther distances downtail, so the exact length of the Mars tail still awaits observational confirmation.

For Venus, where the nominal Parker spiral angle is smaller (i.e., the IMF is more radial), the $\pm Y$ asymmetry of magnetic flux in the tail is significantly larger than at Mars. Plus, the relatively larger solar EUV flux at Venus results in a larger ionospheric thermal plasma pressure, which prevents the draped IMF from penetrating so close to the planet. Both of these factors lead to the tail lobe asymmetry favoring the $+Y$ duskside lobe, and so the magnetotail current sheet at Venus is usually shifted to $-\mathrm{Y}$ dawn side of the midnight meridian.

This shift of magnetotail lobe topology and current sheet location is independent of the solar wind, IMF, and crustal field conditions at Mars. For these simulations, it is the solar EUV flux that controls the location of the tail current sheet.

An interesting comparison can be made with Harnett and Winglee [2005], who conducted an MHD simulation with no crustal fields and a purely By upstream IMF boundary condition. In their results (see their Figure 2), the tail is symmetric. This is expected for the imposed $\mathrm{Bx}=0 \mathrm{IMF}$ condition. In addition, that run had an inner boundary set at $300 \mathrm{~km}$ with a uniform $\mathrm{O}_{2}^{+}$density of $100 \mathrm{~cm}^{-3}$ across the dayside $\left(10 \mathrm{~cm}^{-3}\right.$ on the nightside), which contributes to the symmetry of the result. The results of the present study show that the ionospheric influence of the tail geometry occurs below this altitude, so a shift is not expected in the Harnett and Winglee [2005] simulation results.

This does not exclude the possibility of significant control of current sheet location from these other factors. Certainly, the IMF clock (Y-Z plane) angle will rotate the entire tail configuration relative to Mars [e.g., Luhmann et al., 1992; Modolo et al., 2012]. The IMF azimuthal (X-Y plane) angle will also play a role, as this parameter sets the necessity for an Sshaped topology on the dawn side of the planet, and could make it more like Venus [e.g., Nagy et al., 1990]. The crustal fields have been shown to have a substantial influence on magnetotail topology and planetary ion escape [e.g., Harnett and Winglee, 2005; Fang et al., 2010; Edberg et al., 2011; Li et al., 2013; Ma et al., 2014; Luhmann et al., 2015; Brecht et al., 2016]. Any one of these factors could have a larger influence than that of solar EUV on the location of the tail current sheet. It is left for future studies to examine the relative contribution of all of these factors on the Mars space environment. This study isolated the influence of solar EUV by simplifying the problem and focusing the parameter investigation to a single variable.

While this study does not rule out the influence of crustal fields on the Mars magnetotail structure, it does, however, call into question the finding of Luhmann et al. [2015] that much of the tail lobe magnetic flux is open rather than draped. In their analysis of magnetic field traces similar to those done here, one conclusion of the Luhmann et al. [2015] study was that MHD simulations reveal that most of the lobe magnetic field directly behind the planet is connected to the planet, even when the strong crustal field region is located on the dayside. This study shows a similar finding; a large fraction of the magnetic flux directly behind the planet is open rather than 
draped. Even though the crustal field source was omitted from our simulations, the magnetic field vector in the first true grid cell above the inner boundary is allowed to have a nonzero vertical component, which means that a field line trace through this grid cell will contact the inner boundary surface and be classified as open rather than draped. When draped IMF field lines reach this low of an altitude, the influence on the tail is essentially the same as if it were connected to a planetary crustal field source, but the physics is different. In the Luhmann et al. [2015] case, magnetic reconnection has tied the IMF field line to the planet, making it behave differently than the draped IMF field lines that are slipping past the planet. In the case presented here, it is the ionosphere that holds back the IMF field line from slipping quickly past the planet.

A comment should be made comparing the subsolar boundaries in Figure 8 with observed bow shock and magnetic pile-up boundary locations near Mars. For example, Vignes et al. [2000] used Mars Global Surveyor (MGS) data and found the subsolar bow shock to be at $1.64 \pm$ $0.08 \mathrm{R}_{\mathrm{M}}$, which is close to the solar maximum bow shock locations from the model in Figures $8 \mathrm{a}$ and 8b. From Phobos-2 data, Zhang et al. [1991] found subsolar magnetic pileup region topside altitude of $\sim 550 \mathrm{~km}\left(\sim 1.16 \mathrm{R}_{\mathrm{M}}\right)$, close to the modeled location. Vignes et al. [2000], however, reported a magnetic pileup boundary of $1.29 \pm 0.04 \mathrm{R}_{\mathrm{M}}$, which is a bit farther out than that from the model. Using both Phobos-2 and MGS data, Trotignon et al. [2006] did separate fits to the dayside and nightside boundaries, finding that the subsolar locations move closer to Mars when the fitting procedure is split in this way. Using Mars Express data, Dubinin et al. [2008] found an average subsolar magnetic pileup location of $1.2 \mathrm{R}_{\mathrm{M}}$, which is much closer to the modeled location. This discrepancy is expected given the lack of crustal fields in the simulation setup. In general, the model reproduces the Mars space environment boundaries rather well [cf., Ma et al., 2004; Dong et al., 2015b].

A final comment about this asymmetry is that the coordinate system of this asymmetry and current sheet shift is not well defined by either MSO (Mars Solar Orbital) or MSE (Mars Solar Electric) coordinate systems. It does not have to match MSO because, if the IMF is rotated out of alignment with the ecliptic plane, then the tail lobes and current sheet with also rotate with it. However, it also does not match MSE because this coordinate system aligns the solar wind motional electric field with the $+\mathrm{Z}$ axis by rotating the $\mathrm{Y}-\mathrm{Z}$ axes about the $\mathrm{X}$ axis (i.e., a rotation rather than a mirror-like flip). That is, for a toward-sector IMF orientation, the motional electric field points in the $-\mathrm{Z} \mathrm{MSO}$ direction and the transformation to MSE rotates this by $180^{\circ}$, thereby shifting what was on the dawn side over to the dusk side. So, this effect is not seen clearly in observations because the two standard coordinate systems, MSO and MSE, could potentially average together results from the dawn and dusk sides. So, a different coordinate system, with the IMF aligned with the X-Y plane, should be used to sort the data to confirm this expected influence, as predicted by the analysis of these numerical simulation results. A version of such a coordinate system and data filtering/sorting technique was used by McComas et al. [1986] when they conducted their tail current sheet location study for Venus. 


\section{Conclusion}

Multifluid MHD simulations were conducted with the BATS-R-US code to explore the influence of solar EUV on the magnetic topology of the Mars tail. In particular, the study examined the Y location of the tail current sheet. Observational and numerical studies had revealed the existence of an offset of the tail current sheet away from the midnight meridian. At Venus, this shift is in the $-\mathrm{Y}$ direction, towards dawn. At Mars, the evidence was mixed.

Mars at solar maximum is like the Venus scenario, with the current sheet shifted to the Y direction. At solar minimum, though, the current sheet is shifted in the other direction, to $+Y$. Seasonal-level changes to solar EUV had only a small influence on the location of the current sheet and did not cause a full-scale switch of the location across the midnight meridian.

The physical mechanism controlling this process is the magnetization of the Martian dayside ionosphere. At solar maximum, the draped IMF field lines slip past Mars faster than they do at solar minimum. The induced magnetotail is then Venus-like and the magnetic field collects in the duskside lobe, shifting the current sheet towards dawn. At solar minimum, the ionosphere is weaker and the MPR extends deeper into it. This greatly slows the passage of some magnetic flux from the dayside to the nightside. The $\mathrm{S}$-shape curvature of the field lines on the dawnside becomes greatly elongated, which builds up magnetic field in the dawn lobe as it makes its way back to the dayside ionosphere. This buildup did not occur at solar maximum because much of the magnetic flux slipped relatively quickly around the planet.

Note that all of these simulations used identical upstream solar wind and IMF conditions, and all of them omitted the Mars crustal field sources. The change in magnetotail lobe structure and current sheet location in this study, therefore, is entirely because of the change in solar EUV intensity. While these other factors should be added back into the investigation for future parameter studies, the conclusion of this study is that the ionosphere exerts significant control over the induced magnetotail at Mars.

\section{Acknowledgments and Data}

The authors thank NASA for supporting this work, particularly under grants NNX16AQ04G, NNX13AF26G, and NNX11AD80G. Model results are available upon request to the authors. Simulations for this work were performed at the University of Michigan Advanced Research Computing Center and the NASA High-End Computer Center, in particular the Flux and Pleiades machines at these two facilities, respectively.

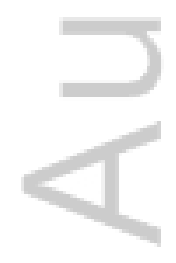




\section{References}

Barabash, S., A. Fedorov, R. Lundin, and J.-A. Sauvaud (2007), Martian atmospheric erosion rates, Science, 315, 501-503, doi:10.1126/ science.1134358.

Bertucci, C., F. Duru, N. Edberg, M. Fraenz, C. Martinecz, K. Szego, and O. Vaisberg (2011), The induced magnetospheres of Mars, Venus, and Titan, Space Sci. Rev., 162, 113-171,

doi: $10.1007 / \mathrm{s} 11214-011-9845-1$.

Bougher, S., D. Pawlowski, J. Bell, S. Nelli, T. McDunn, J. Murphy, M. Chizek, and A. Ridley (2015), Mars global ionosphere-thermosphere model: Solar cycle, seasonal, and diurnal

= variations of the Mars upper atmosphere, J. Geophys. Res. Planets, 120, 311-342, doi:10.1002/2014JE004715.

Brain, D. A., D. L. Mitchell, and J. S. Halekas (2006), The magnetic draping direction at Mars from April 1999 through August 2004, Icarus, 182, 464-473, doi: 10.1016/j.icarus.2005.09.023.

Brecht, S. H., S. A. Ledvina, and S. W. Bougher (2016), Ionospheric loss from Mars as predicted by hybrid particle simulations, J. Geophys. Res. Space Physics, 121, doi:10.1002/2016JA022548.

Carlsson, E., et al., (2006), Mass composition of the escaping plasma at Mars. Icarus 182, 320328.

Carlsson, E., D. Brain, J. Luhmann, S. Barabash, A. Grigoriev, H. Nilsson, and R. Lundin (2008), Influence of IMF draping direction and crustal magnetic field location on Martian ion beams, Planet. Space Sci., 56, 861-867, doi: 10.1016/j.pss.2007.12.016.

DiBraccio, G. A., et al. (2015), Magnetotail dynamics at Mars: Initial MAVEN observations, Geophys. Res. Lett., 42, 8828-8837, doi:10.1002/2015GL065248.

Dong, C., S. W. Bougher, Y. Ma, G. Toth, A. F. Nagy, and D. Najib (2014), Solar wind interaction with Mars upper atmosphere: Results from the one-way coupling between the multifluid MHD model and the MTGCM model, Geophys. Res. Lett., 41, 2708-2715, doi:10.1002/2014GL059515.

Dong, C., S. W. Bougher, Y. Ma, G. Toth, Y. Lee, A. F. Nagy, V. Tenishev, D. J. Pawlowski, M. R. Combi, and D. Najib (2015a), Solar wind interaction with the Martian upper atmosphere: Crustal field orientation, solar cycle, and seasonal variations, J. Geophys. Res. Space Physics, 120, 7857-7872, doi:10.1002/2015JA020990.

Dong, C., et al. (2015b), Multifluid MHD study of the solar wind interaction with Mars' upper atmosphere during the 2015 March 8th ICME event, Geophys. Res. Lett., 42, 9103-9112, doi:10.1002/2015GL065944.

This article is protected by copyright. All rights reserved. 
Dubinin, E., et al. (2006), Electric fields within the Martian magnetosphere and ion extraction: ASPERA-3 observations, Icarus, 182, 337 - 342, doi:10.1016/j.icarus.2005.05.022.

Dubinin, E., et al. (2008), Structure and dynamics of the solar wind/ionosphere interface on Mars: MEX-ASPERA-3 and MEX-MARSIS observations, Geophys. Res. Lett., 35 , L11103, doi:10.1029/2008GL033730.

Dubinin, E., M. Fraenz, A. Fedorov, R. Lundin, N. Edberg, F. Duru, and O. Vaisberg (2011), Ion energization and escape on Mars and Venus, Space Sci. Rev., 162, 173-211, doi: 10.1007/s11214-011-9831-7.

Dubinin, E., and M. Fraenz (2015), Magnetotails of Mars and Venus, in Magnetotails in the Solar System, Geophysical Monograph Series, 207, 43-59, American Geophysical Union, John Wiley and Sons.

Edberg, N. J. T., et al. (2011), Atmospheric erosion of Venus during stormy space weather, $J$. Geophys. Res., 116, A09308, doi:10.1029/2011JA016749.

Fang, X., M. W. Liemohn, A. F. Nagy, Y. Ma, D. L. De Zeeuw, J. U. Kozyra, and T. Zurbuchen (2008), Pickup oxygen ion distribution around Mars, J. Geophys. Res., 113, A02210, doi: 10.1029/2007JA012736.

Fang, X., M. W. Liemohn, A. F. Nagy, J. G. Luhmann, and Y. Ma (2010), On the effect of the Martian crustal magnetic field on atmospheric erosion, Icarus, 206, 130, doi: 10.1016/j.icarus.2009.01.012.

Fang, X., Y. Ma, D. Brain, Y. Dong, and R. Lillis (2015), Control of Mars global atmospheric loss by the continuous rotation of the crustal magnetic field: A time-dependent MHD study, J. Geophys. Res. Space Physics, 120, 10,926-10,944, doi:10.1002/2015JA021605.

T. I. Gombosi, G. Toth, D. L. De Zeeuw, K. C. Hansen, K. Kabin, K. G. Powell (2002), SemiRelativistic Magnetohydrodynamics and Physics-Based Convergence Acceleration, Journal of Computational Physics, 177, 176-205.

Halekas, J. S., D. A. Brain, R. J. Lillis, M. O. Fillingim, D. L. Mitchell, and R. P. Lin (2006), Current sheets at low altitudes in the Martian magnetotail, Geophys. Res. Lett., 33, L13101, doi:10.1029/2006GL026229.

Harnett, E. M., and R. M. Winglee (2005), Three-dimensional fluid simulations of plasma asymmetries in the Martian magnetotail caused by the magnetic anomalies, J. Geophys. Res., 110, A07226, doi:10.1029/2003JA010315.

Lee, Y., M. R. Combi, V. Tenishev, S. W. Bougher, and R. J. Lillis (2015), Hot oxygen corona at Mars and the photochemical escape of oxygen - Improved description of the thermosphere, ionosphere and exosphere \}, J. Geophys. Res. Planets, 120, 1880-1892, doi:10.1002/2015JE004890.

This article is protected by copyright. All rights reserved. 
Liemohn, M. W., Y. Ma, R. A. Frahm, X. Fang, J. U. Kozyra, A. F. Nagy, J. D. Winningham, J. R. Sharber, S. Barabash, and R. Lundin (2006), Mars global MHD predictions of magnetic connectivity between the dayside ionosphere and the magnetospheric flanks, Space Sci. Rev., 126, 63-76.

Li, L., L. Xie, Y. Zhang, T. Liu (2013), Model investigation of current system and influence of the crustal fields on the large scale structure of current sheets at Mars, Planet. Space Sci., 86, 80-85, doi: 10.1016/j.pss.2013.07.004.

Luhmann, J. G., and L. H. Brace (1991), Near-Mars space, Rev. Geophys., 29, 121.

Luhmann, J. G., C. T. Russell, L. H. Brace, and O. L. Vaisberg (1992), Ch. 31, The intrinsic magnetic field and solar wind interaction of Mars, in Mars, edited by H. H. Kieffer, B. M. Jakosky, C. W. Snyder, and M. S. Matthews, p. 1054, Univ. of Arizona Press, Tucson.

Luhmann, J. G., et al. (2015), Implications of MAVEN Mars near-wake measurements and models, Geophys. Res. Lett., 42, 9087-9094, doi:10.1002/2015GL066122.

Ma, Y., A. F. Nagy, K. C. Hansen, D. L. DeZeeuw, T. I. Gombosi, and K. G. Powell (2002), Three-dimensional multispecies MHD studies of the solar wind interaction with Mars in the presence of crustal fields, J. Geophys. Res., 107(A10), 1282, doi:10.1029/2002JA009293.

Ma, Y., A. F. Nagy, I. V. Sokolov, and K. C. Hansen (2004), Three-dimensional, multispecies, high spatial resolution MHDstudies of the solar wind interaction with Mars, J. Geophys. Res., 109, A07211, doi:10.1029/2003JA010367.

Ma,Y. J., A. F. Nagy, C. T. Russell,R. J. Strangeway, H. Y.Wei, andG.Toth (2013),Aglobalmultispecies single-fluid MHD study of the plasma interaction around Venus, J. Geophys. Res. Space Physics, 118, 321-330, doi:10.1029/2012JA018265.

Ma, Y., X. Fang, C. T. Russell, A. F. Nagy, G. Toth, J. G. Luhmann, D. A. Brain, and C. Dong (2014), Effects of crustal field rotation on the solar wind plasma interaction with Mars, Geophys. Res. Lett., 41, 6563-6569, doi:10.1002/2014GL060785.

McComas, D. J., H. E. Spence, C. T. Russell, and M. A. Saunders (1986), the average magnetic field draping and consistent plasma properties of the Venus magnetotail, J. Geophys. Res., 91, 7939-7953.

Modolo, R., G. M. Chanteur, and E. Dubinin (2012), Dynamic Martian magnetosphere: Transient twist induced by a rotation of the IMF, Geophys. Res. Lett., 39, L01106, doi:10.1029/2011GL049895.

Nagy, A. F., T. I. Gombosi, K. Szego, R. Z. Sagdeev, V. D. Shapiro, and V. I. Shevchenko (1990), Venus mantle-Mars planetosphere: what are the similarities and differences?, Geophys. Res. Lett., 17, 865.

This article is protected by copyright. All rights reserved. 
Nagy, A., et al. (2004), The plasma environment of Mars, in Mars' Magnetism and Its Interaction With the Solar Wind, edited by D.Winterhalter, M. Acuna, and A. Zakharov, pp. 33-114, Springer, Netherlands.

Najib, D., A. F. Nagy, G. Toth, and Y. Ma (2011), Three-dimensional, multifluid, high spatial resolution MHD model studies of the solar wind interaction with Mars, J. Geophys. Res., $\_116($ A5), doi:10.1029/2010ja016272.

Riedler, W., K. Schingenschuh, H. Lichtenegger, D. Möhlmann, J. Rustenbach, Ye. Yeroshenko, J. Achache, J. Slavin, J. G. Luhmann, and C. T. Russell (1991), Interaction of the solar wind with the planet Mars: Phobos 2 magnetic field observations, Planet. Space Sci., 39, 75-81, doi: 10.1016/0032-0633(90)90129-X.

Schwingenschuh, K., et al. (1992), The Martian magnetic field environment: induced or dominated by an intrinsic magnetic field?, Adv. Space Res., 12(9), 213-219, doi: 10.1016/0273-1177(92)90333-S.

Trotignon, J. G., C. Mazelle, C. Bertucci, and M. H. Acuña (2006), Martian sock and magnetic pile-up boundary positions and shapes determined from the Phobos 2 and Mars Global Surveyor data sets, Planet. Space Sci., 54, 357-369, doi: 10.1016/j.pss.2006.01.003.

Vignes, D., et al. (2000), The solar wind interaction with Mars: Location and shapes of the bow shock and magnetic pileup boundary from observations of the MAG/ER experiment onboard Mars Global Surveyor, Geophys. Res. Lett., 27, 49.

Xu., S., M. W. Liemohn, C. Dong, D. L. Mitchell, and S. M. Bougher (2016), Pressure and ion composition boundaries at Mars, J. Geophys. Res. Space Physics, 121, 6417-6429, doi: 10.1002/2016JA022644.

Yeroshenko, Ye., W. Riedler, K. Schwingenschuh, J. G. Luhmann, M. Ong, and C. T. Russell (1990), The magnetotail of Mars: Phobos observations, Geophys. Res. Lett., 17, 885-888, doi: 10.1029/GL017i006p00885.

Zhang, T. L., Luhmann, J. G. and C. T. Russell (1991), The Magnetic Barrier at Venus, J. Geophys. Res. 96, 11145-11153.

This article is protected by copyright. All rights reserved. 
(a) Solar Max @ Perihelion

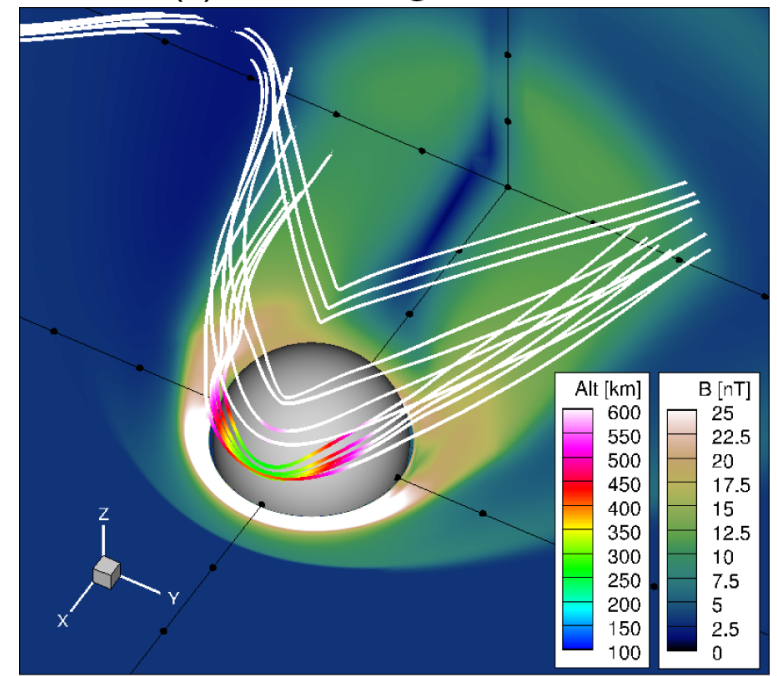

(c) Solar Min @ Perihelion

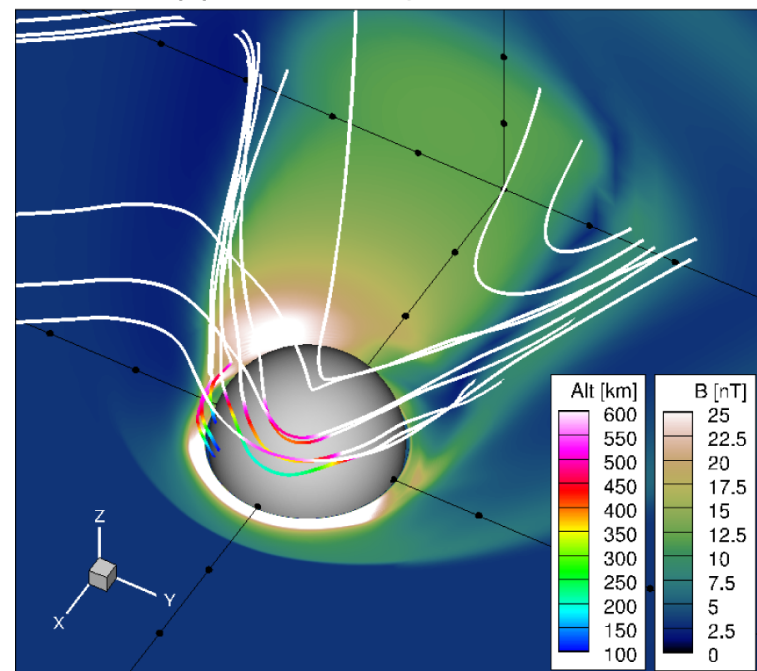

(b) Solar Max @ Aphelion

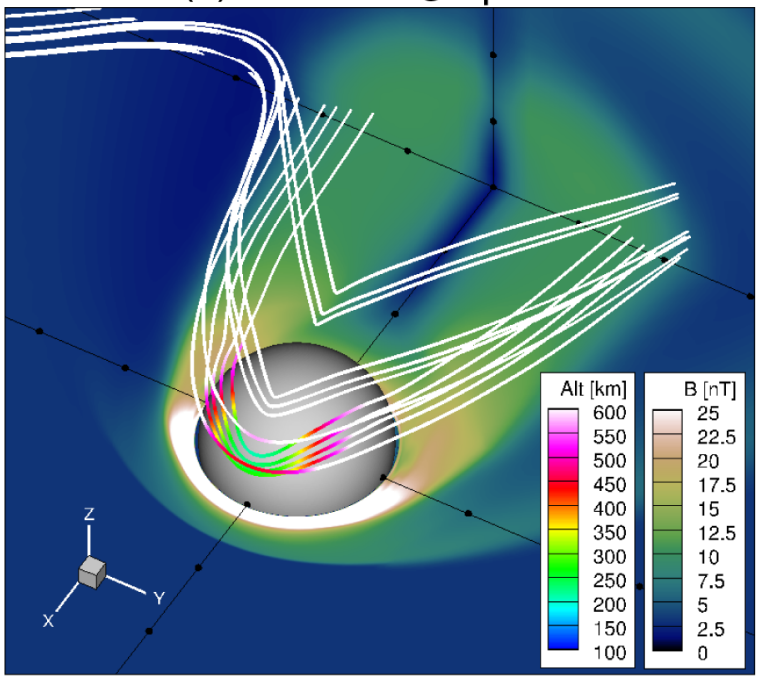

(d) Solar Min @ Aphelion

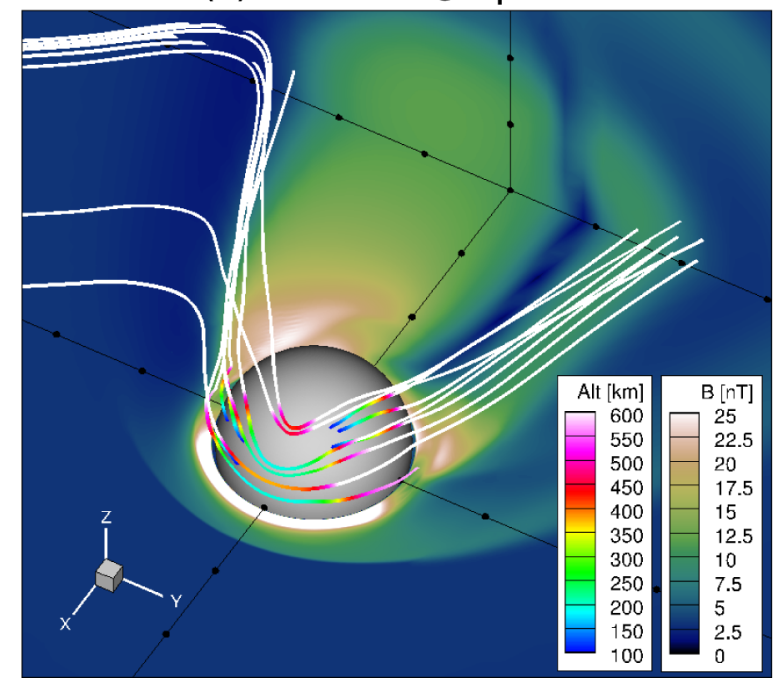

2016JA023707-f01-z-.tif 
(a) Solar Max @ Perihelion

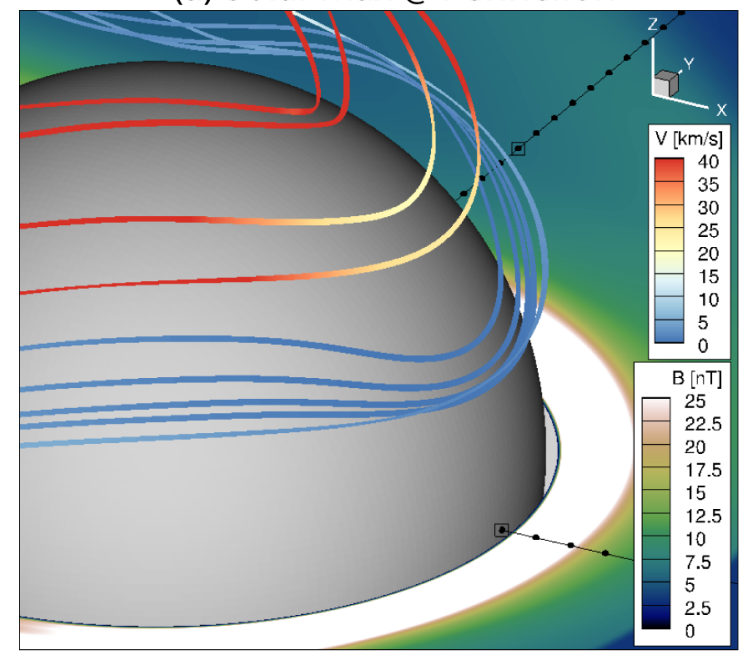

(c) Solar Min @ Perihelion

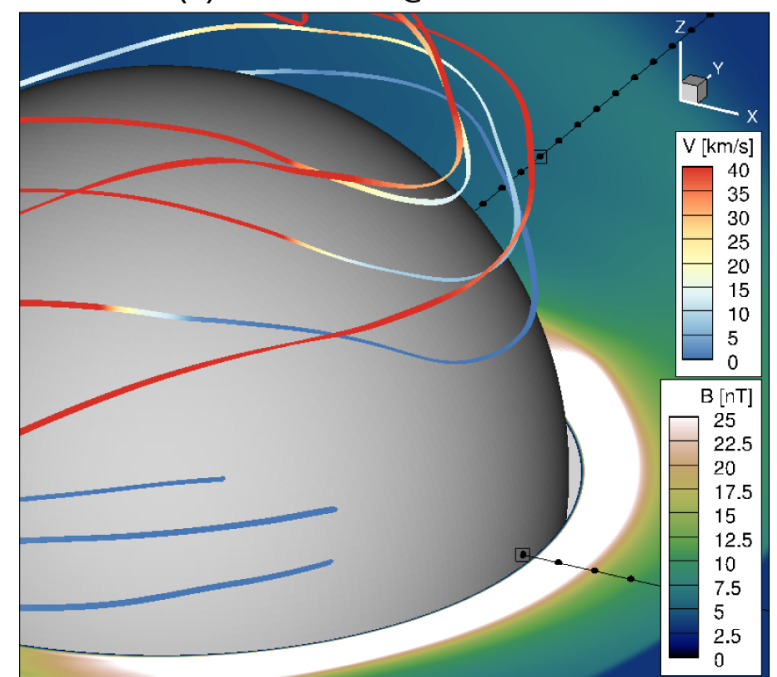

(b) Solar Max @ Aphelion

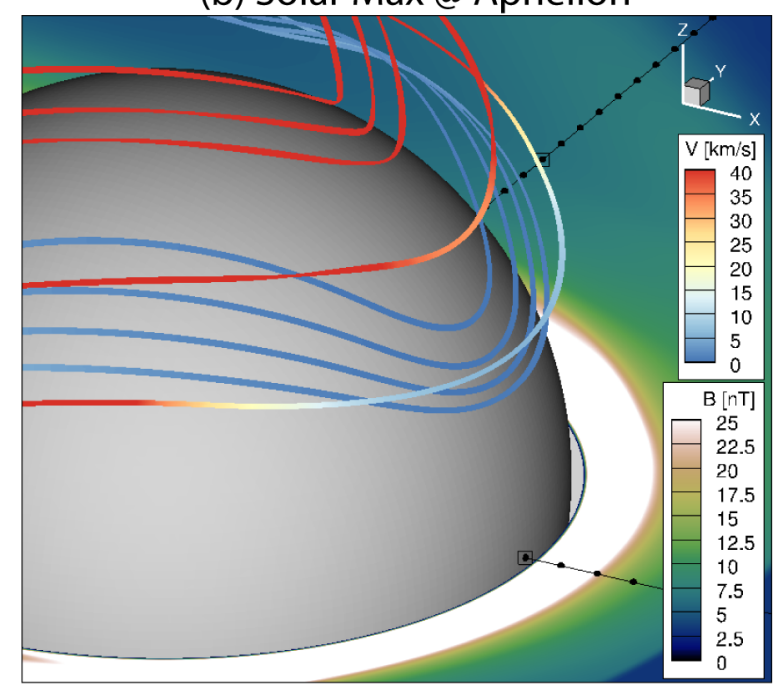

(d) Solar Min @ Aphelion

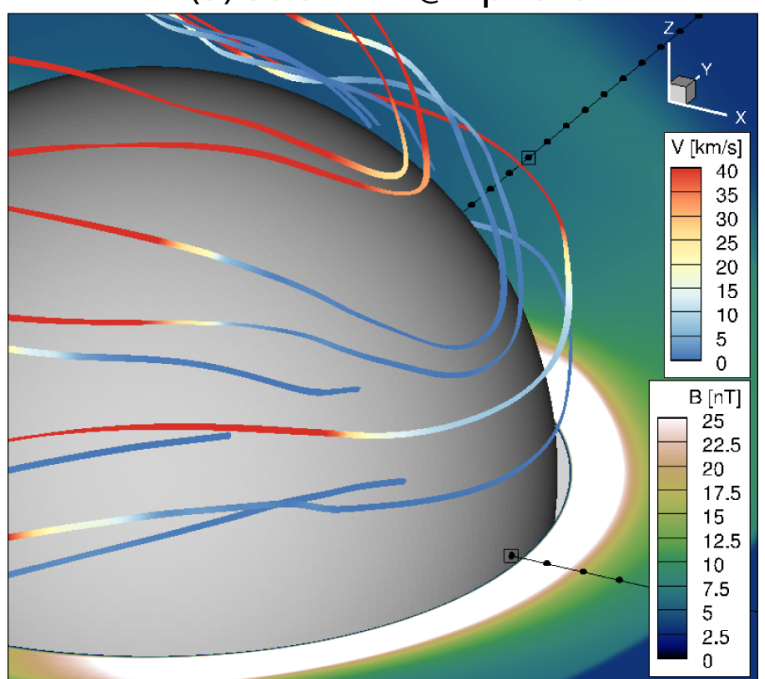

2016JA023707-f02-z-.tif

This article is protected by copyright. All rights reserved. 
(a) Solar Max @ Perihelion

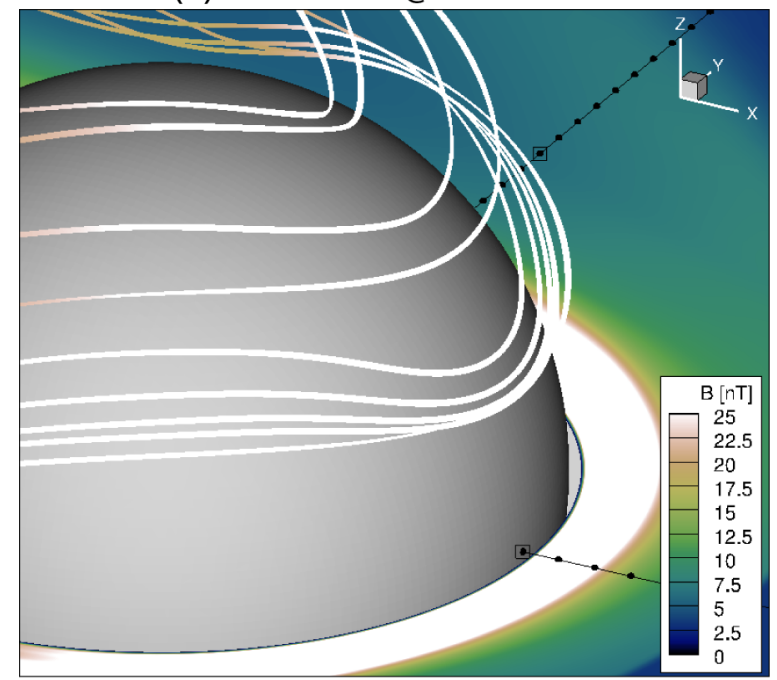

(c) Solar Min @ Perihelion

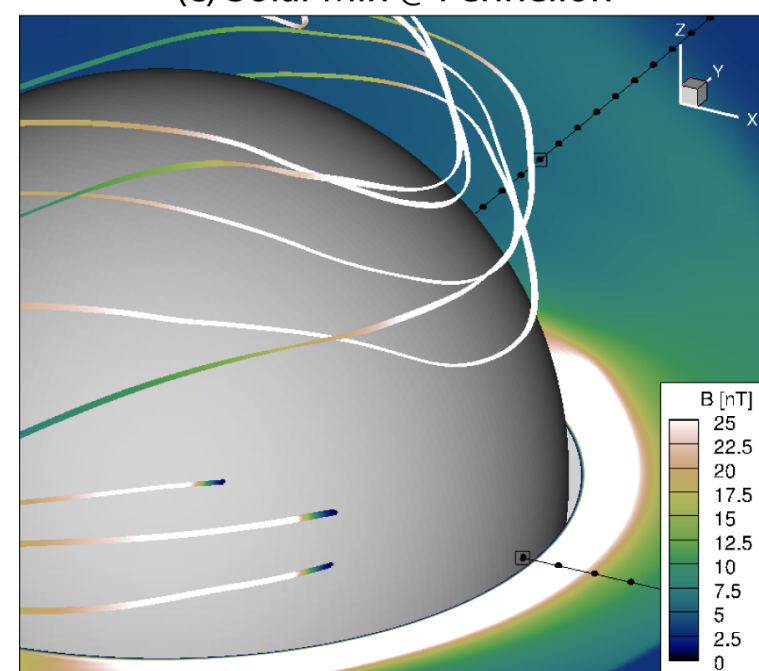

(b) Solar Max @ Aphelion

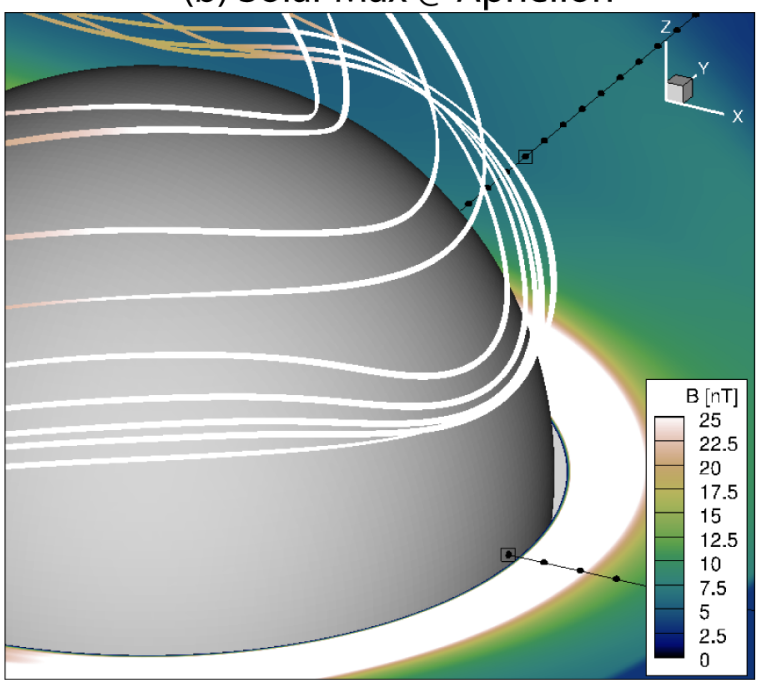

(d) Solar Min @ Aphelion

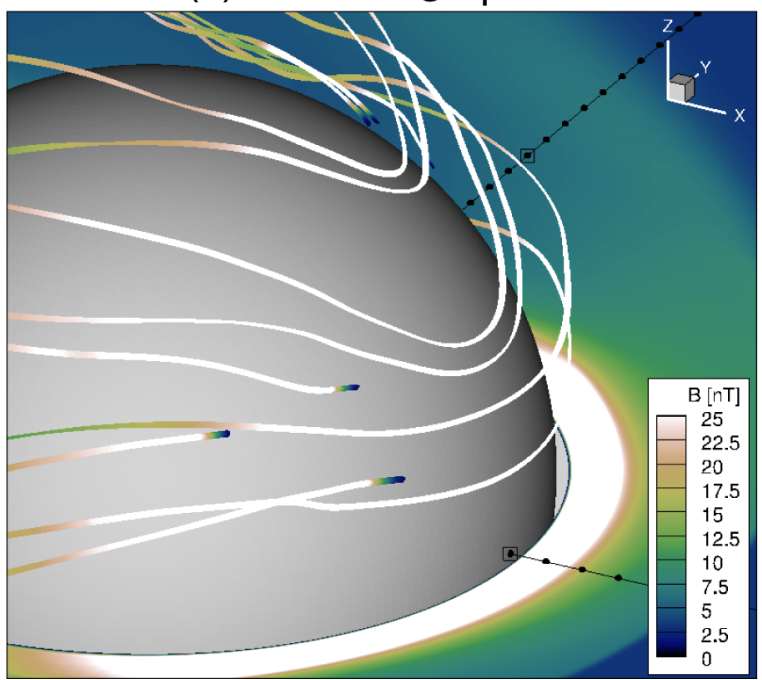

2016JA023707-f03-z-.tif 


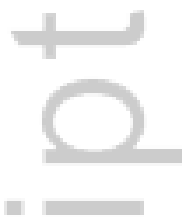

(a) Solar Max @ Perihelion

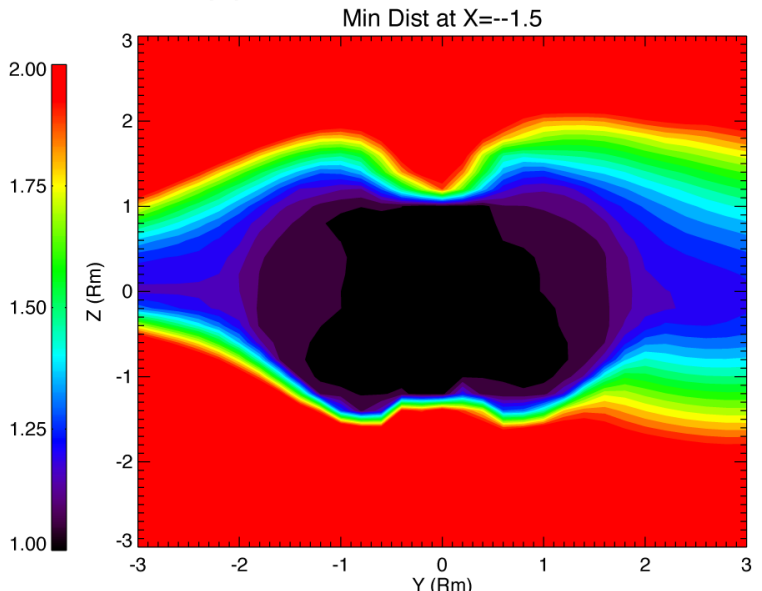

(c) Solar Min @ Perihelion

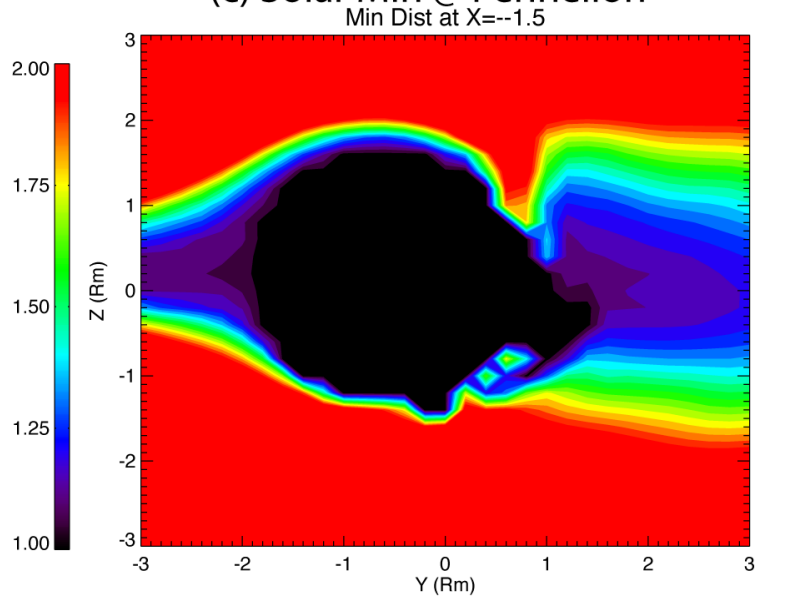

2016JA023707-f04-z-.tif

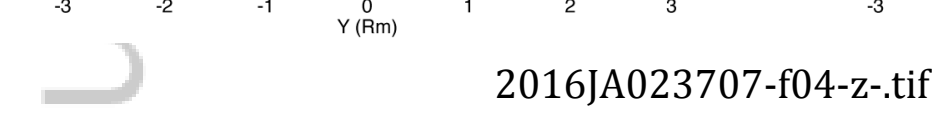

(b) Solar Max @ Aphelion

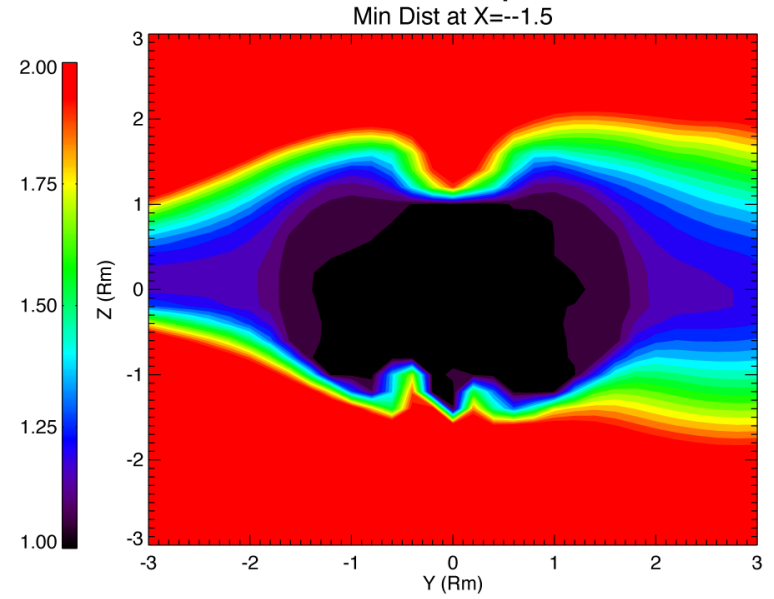

(d) Solar Min @ Aphelion

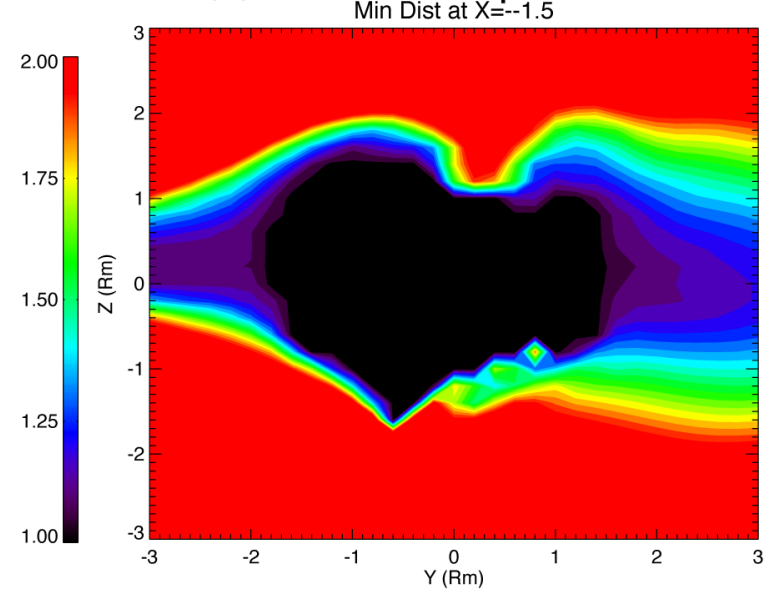

This article is protected by copyright. All rights reserved. 


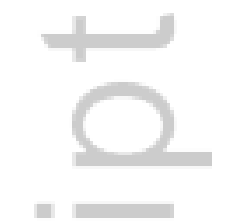

(a) Solar Max @ Perihelion log10(IVI) @ min Alt; X=--1.5

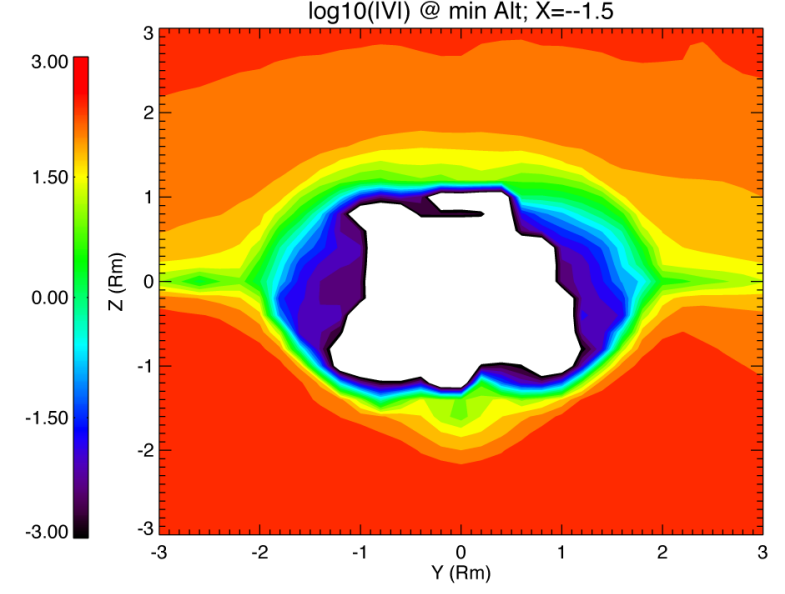

(c) Solar Min @ Perihelion

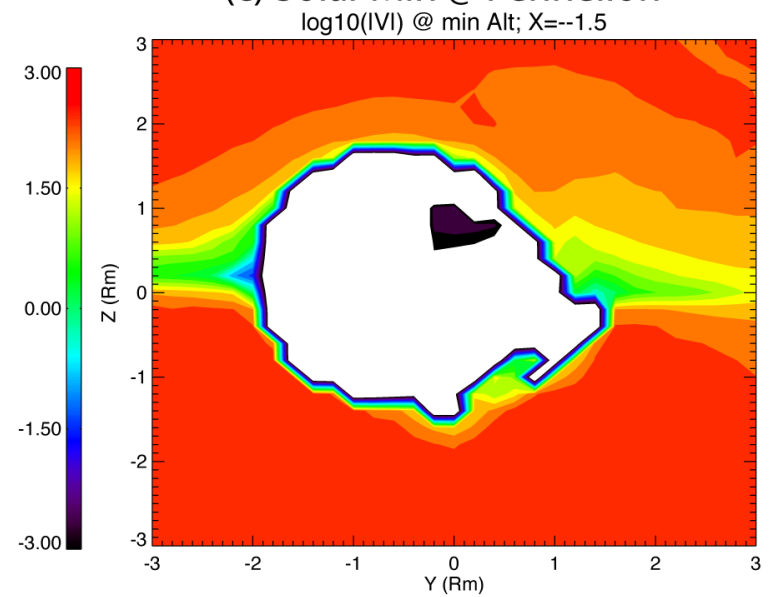

2016JA023707-f05-z-.tif (b) Solar Max @ Aphelion $\log 10(\mathrm{IVI}) @$ min Alt; X=--1.5

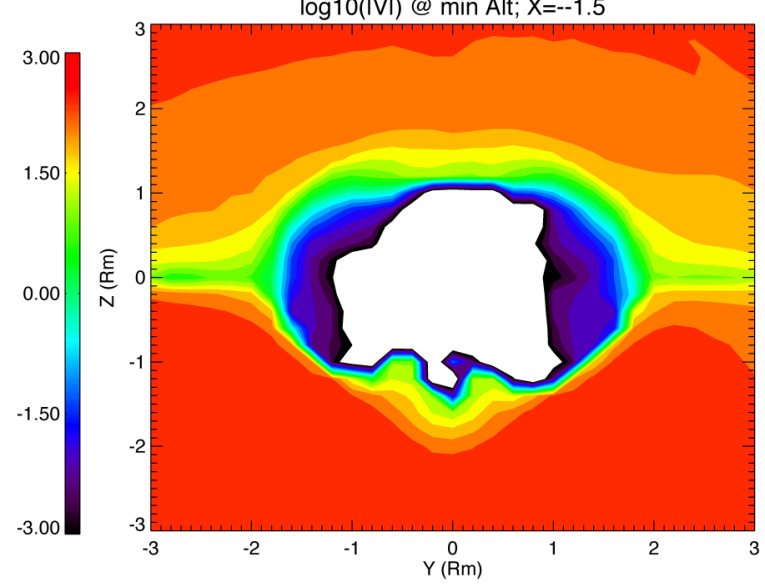

(d) Solar Min @ Aphelion

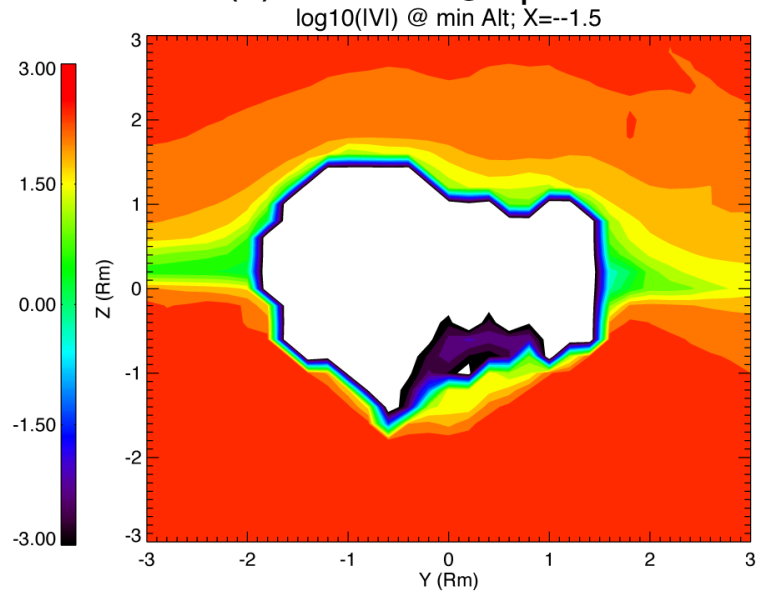

This article is protected by copyright. All rights reserved. 
(a) Solar Max @ Perihelion

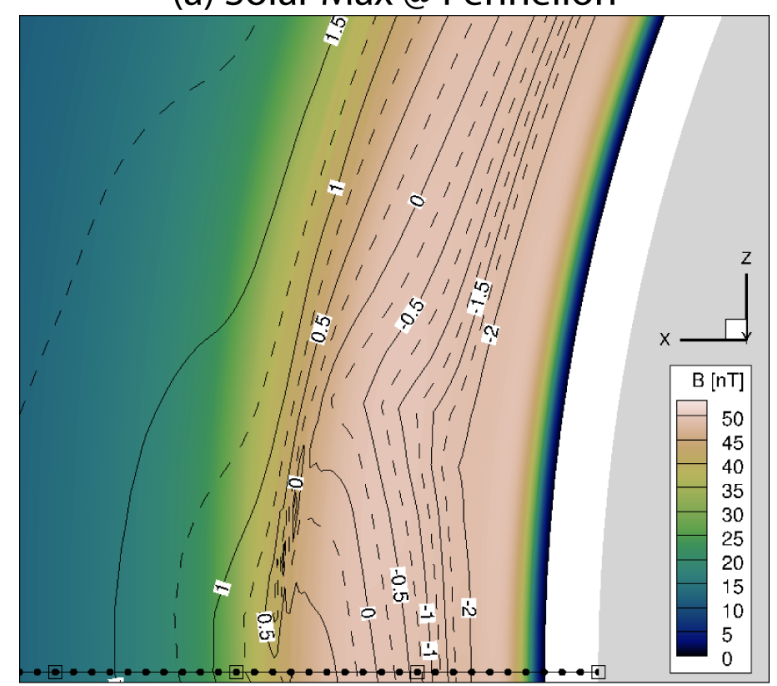

(c) Solar Min @ Perihelion

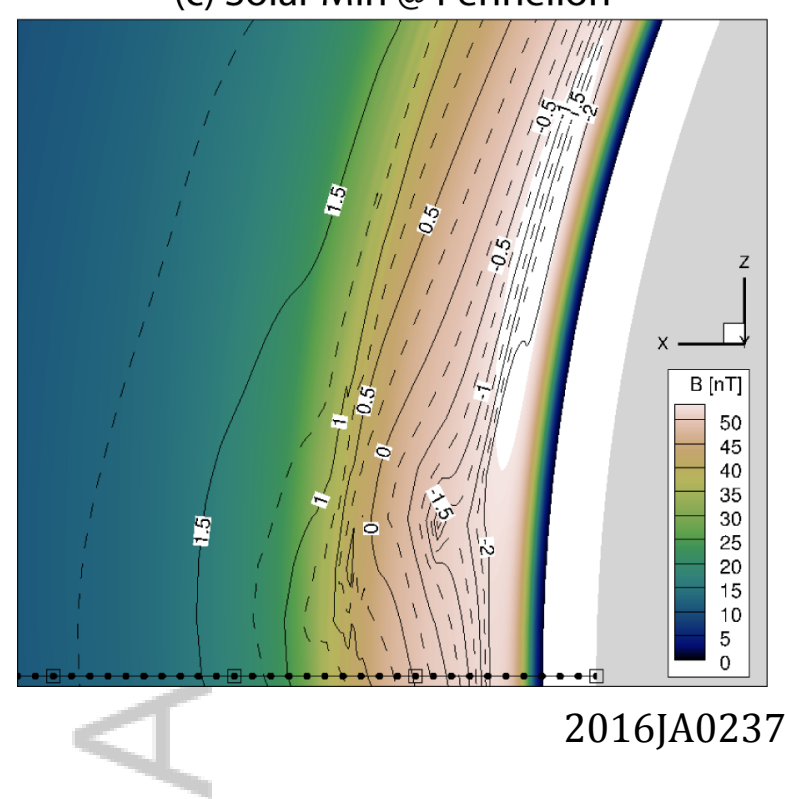

(b) Solar Max @ Aphelion

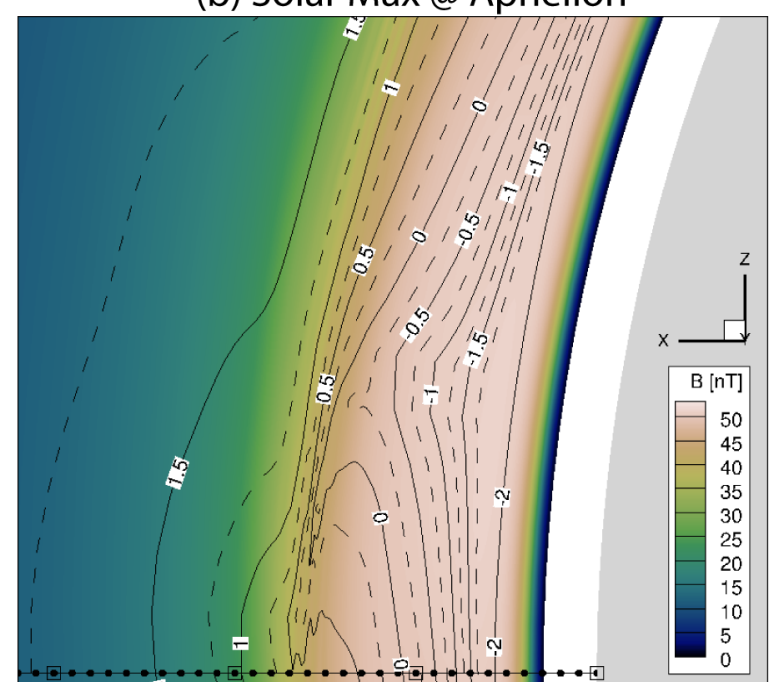

(d) Solar Min @ Aphelion

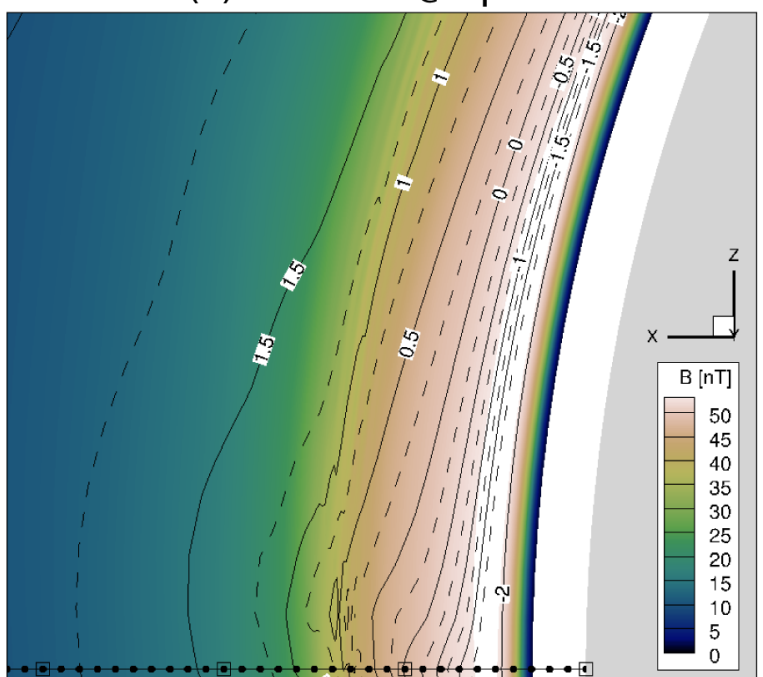

07-f06-z-.tif 
(a) Solar Max @ Perihelion

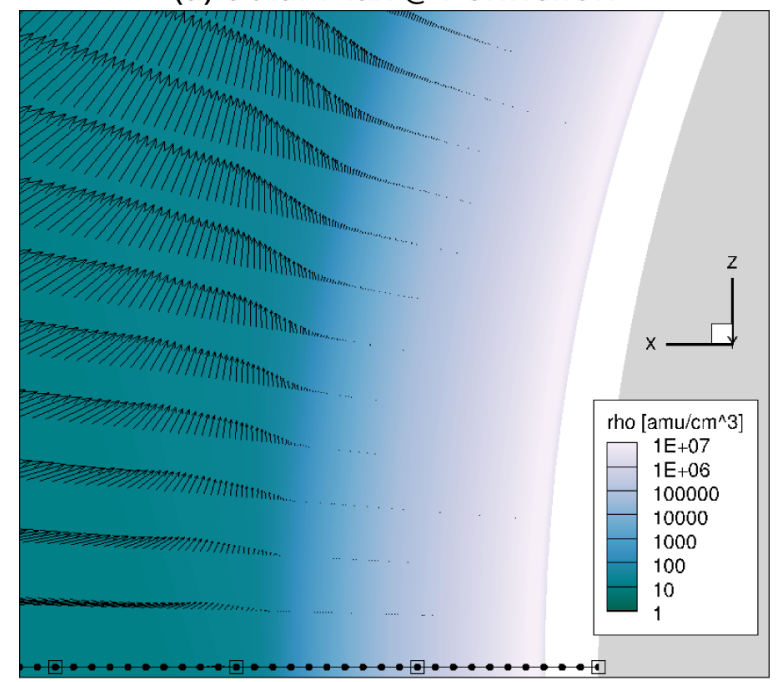

(c) Solar Min @ Perihelion

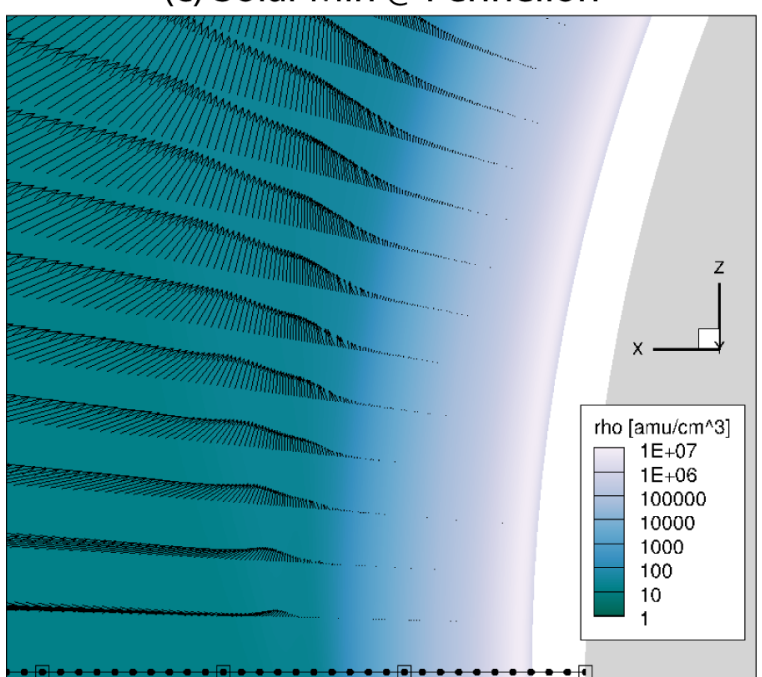

2016JA023707-f07-z-.tif (b) Solar Max @ Aphelion

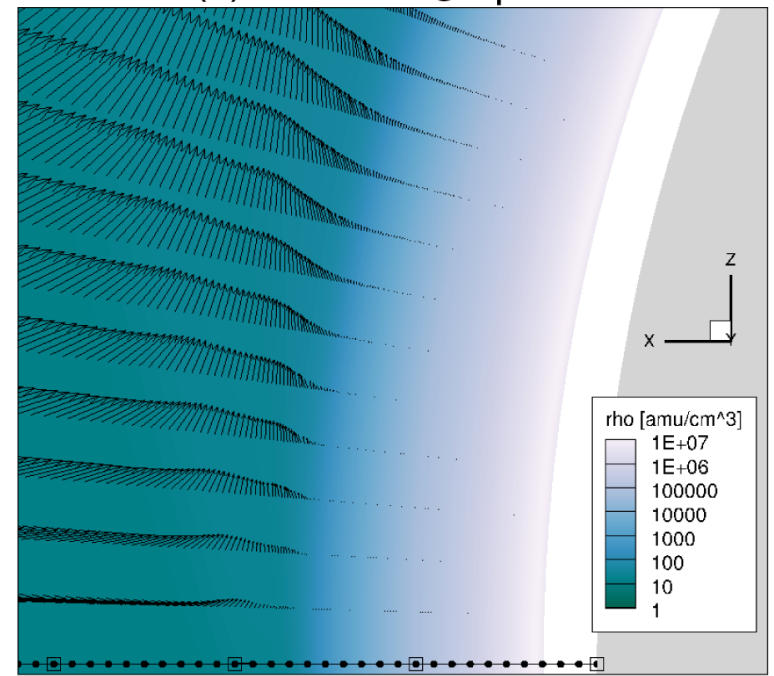

(d) Solar Min @ Aphelion

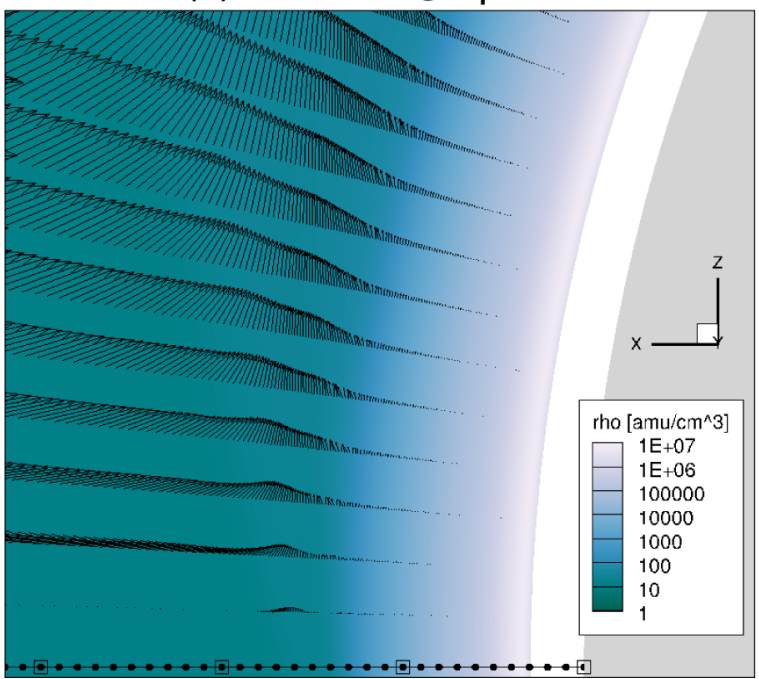


(a) Solar Max @ Perihelion

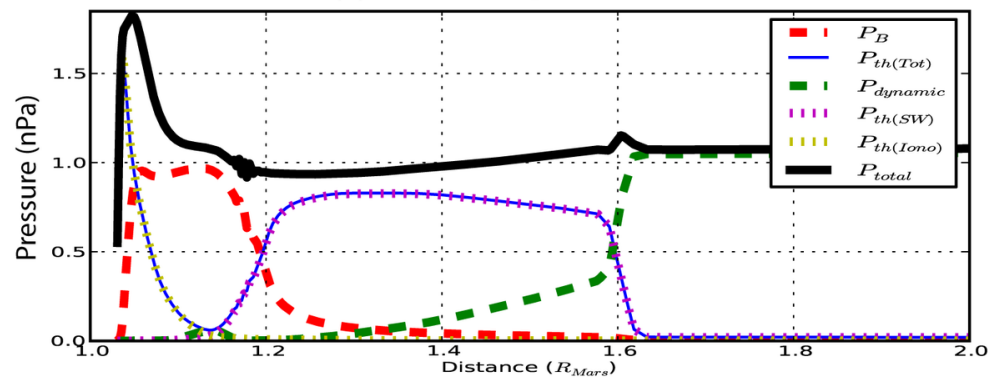

(b) Solar Max @ Aphelion

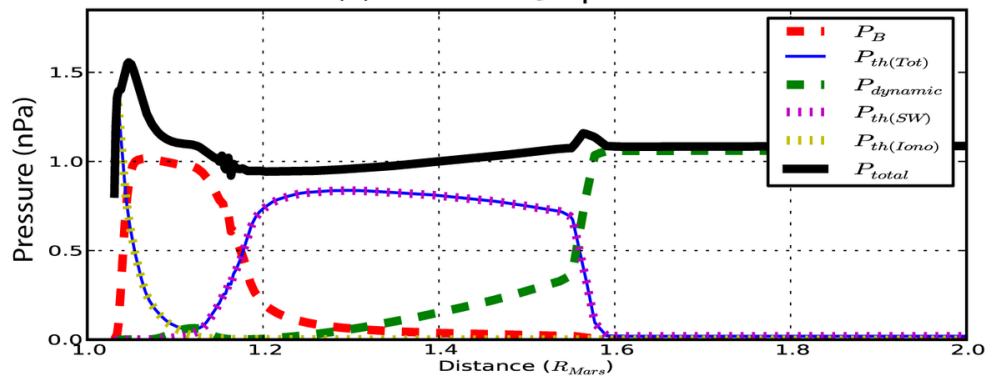

(c) Solar Min @ Perihelion

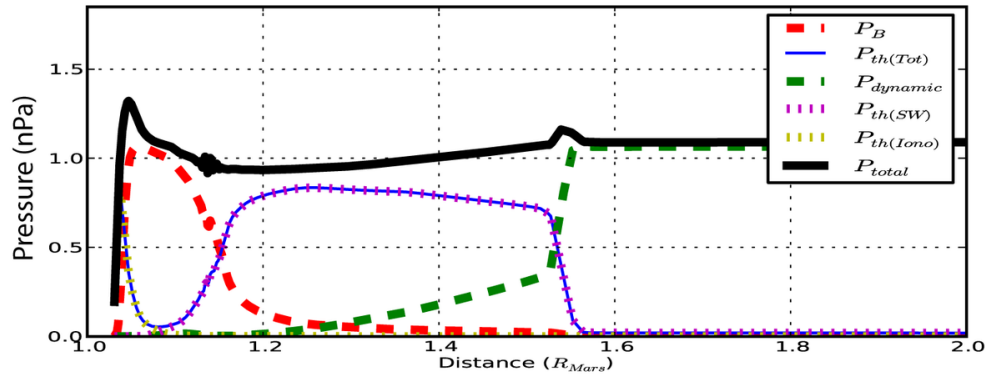

(d) Solar Min @ Aphelion

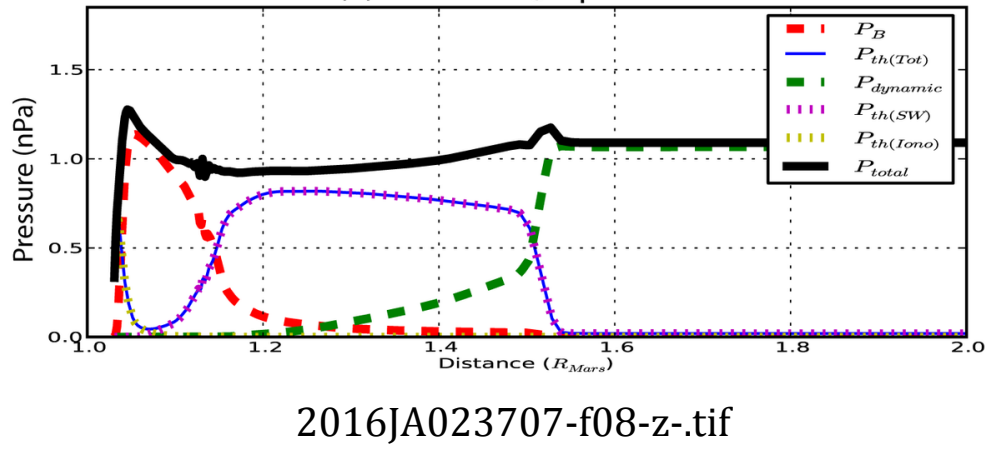

This article is protected by copyright. All rights reserved. 
(a) Solar Max @ Perihelion

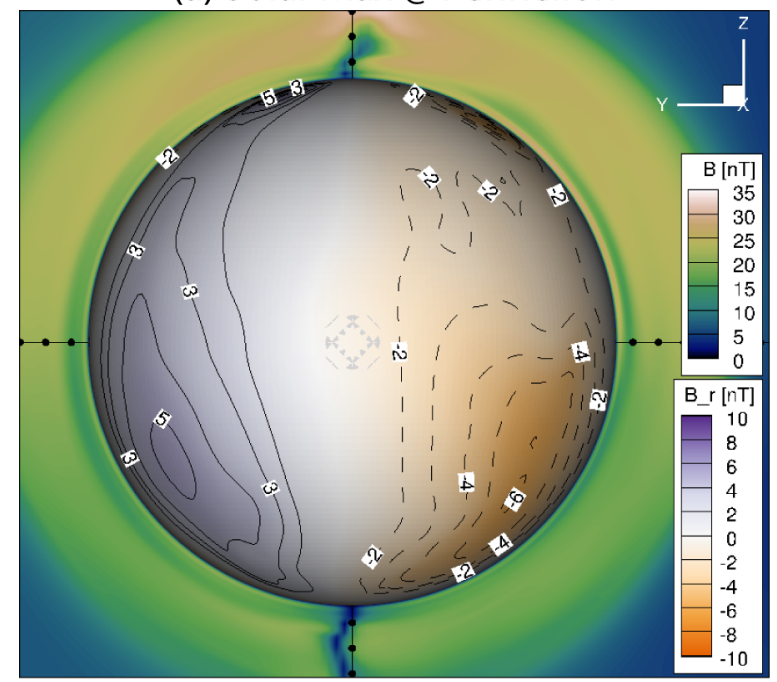

(c) Solar Min @ Perihelion

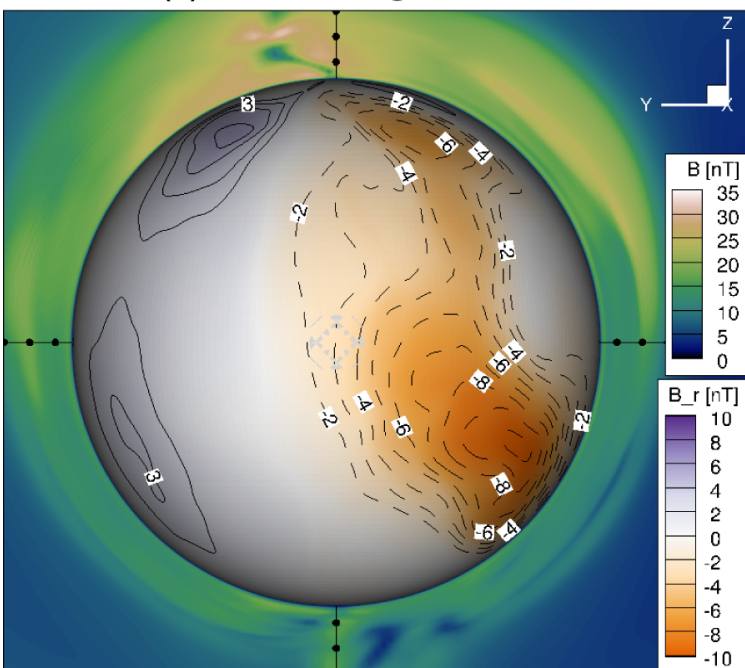

(b) Solar Max @ Aphelion

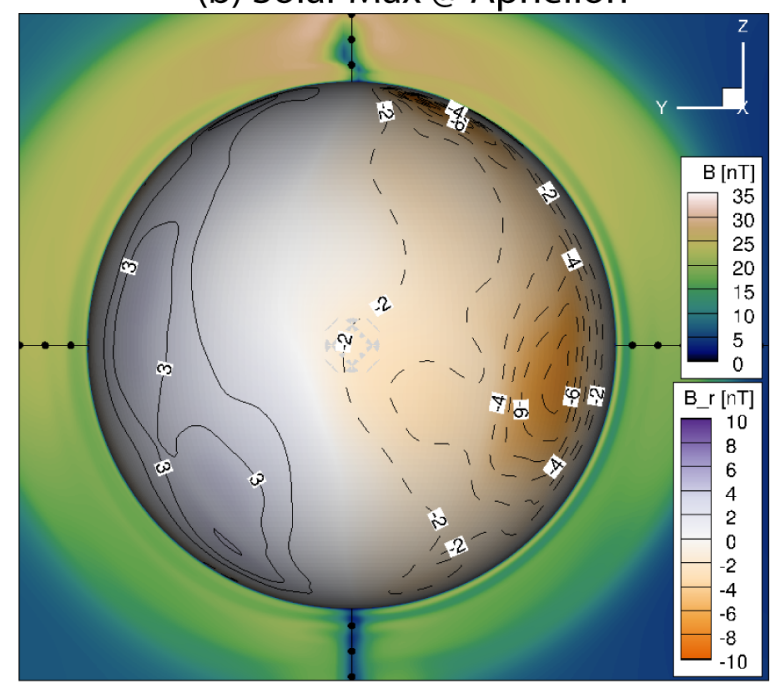

(d) Solar Min @ Aphelion

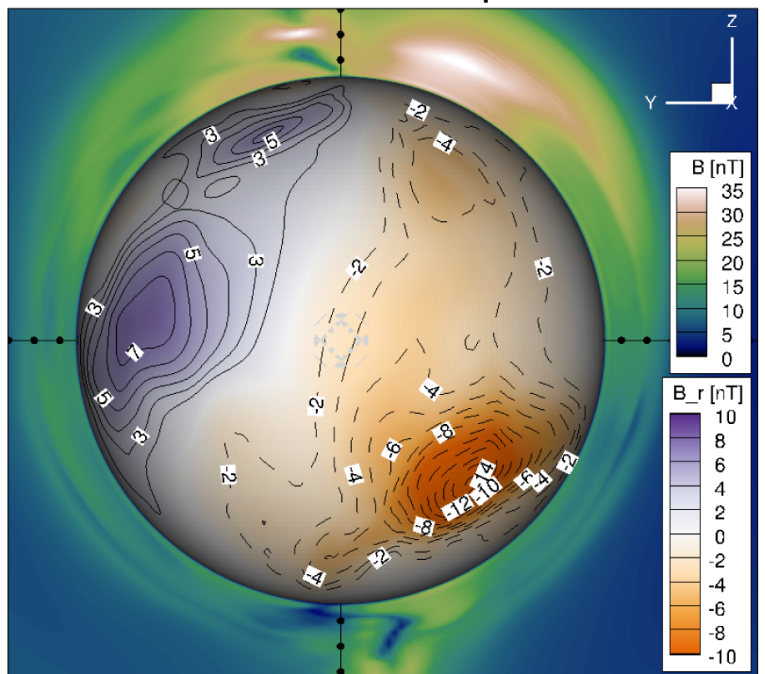

2016JA023707-f09-z-.tif 